\title{
The Prediction of Scattered Broadband Shock-Associated Noise
}

\author{
Steven A. E. Miller* \\ The National Aeronautics and Space Administration
}

\begin{abstract}
A mathematical model is developed for the prediction of scattered broadband shockassociated noise. Model arguments are dependent on the vector Green's function of the linearized Euler equations, steady Reynolds-averaged Navier-Stokes solutions, and the twopoint cross-correlation of the equivalent source. The equivalent source is dependent on steady Reynolds-averaged Navier-Stokes solutions of the jet flow, that capture the nozzle geometry and airframe surface. Contours of the time-averaged streamwise velocity component and turbulent kinetic energy are examined with varying airframe position relative to the nozzle exit. Propagation effects are incorporated by approximating the vector Green's function of the linearized Euler equations. This approximation involves the use of ray theory and an assumption that broadband shock-associated noise is relatively unaffected by the refraction of the jet shear layer. A non-dimensional parameter is proposed that quantifies the changes of the broadband shock-associated noise source with varying jet operating condition and airframe position. Scattered broadband shock-associated noise possesses a second set of broadband lobes that are due to the effect of scattering. Presented predictions demonstrate relatively good agreement compared to a wide variety of measurements.
\end{abstract}

\section{Nomenclature}

$\begin{array}{ll}\text { Symbols } & \text { Description } \\ A_{s}, B_{s}, C_{s} & \text { Prefactor constants } \\ a_{m n} & \text { Anisotropic turbulence coefficients } \\ c & \text { Speed of sound } \\ c_{l}, c_{u}, c_{\tau} & \text { Coefficients of turbulent scales } \\ D & \text { Nozzle exit diameter } \\ D_{j} & \text { Fully expanded diameter } \\ \tilde{D} & \text { Diffraction coefficient } \\ \boldsymbol{E} & \text { Vector from source to airframe edge } \\ f & \text { Frequency } \\ g & \text { Green's function } \\ k & \text { Turbulent kinetic energy or wavenumber } \\ k_{1} & \text { Axial wavenumber of } p_{s} \\ l_{i} & \text { Component of turbulent length scale } \\ M_{d} & \text { Design Mach number } \\ M_{j} & \text { Fully expanded Mach number } \\ p & \text { Pressure } \\ p_{s} & \text { Shock pressure } \\ R & \text { Distance to observer from nozzle exit } \\ \boldsymbol{r} & \text { Vector from source to observer } \\ \boldsymbol{r}_{1} & \text { Vector from airframe edge to observer } \\ S & \text { Spectral density of acoustic pressure } \\ S t & \text { Strouhal number } \\ t & \text { Time } \\ \boldsymbol{u} & \text { Velocity vector } \\ u_{s} & \text { Turbulent velocity scale } \\ v_{g o}^{n} & \text { Velocity component of vector } \\ \boldsymbol{x} & \text { Green's function of LEE } \\ x_{p} & \text { Observer vector } \\ & \text { Axial distance from nozzle } \\ & \text { exit to trailing edge }\end{array}$

$\begin{array}{ll}\boldsymbol{y} & \text { Source vector } \\ y_{p} & \text { Radial distance from nozzle } \\ \boldsymbol{z} & \text { exit to trailing edge } \\ \beta & \text { Vector from source to observer } \\ \Gamma & \text { Wedge angle } \\ \gamma & \text { Non-dimensional parameter } \\ \delta & \text { Ratio of specific heats } \\ \delta_{\eta} & \text { Dirac delta function } \\ \epsilon & \text { Jet spreading rate } \\ \nu & \text { Dissipation } \\ \pi_{g}^{n} & \pi / \beta \\ & \text { Pressure component of vector } \\ \tau & \text { Green's function of LEE } \\ \tau & \text { Density } \\ \tau_{s} & \text { Retarded time } \\ \phi_{o} & \text { Turbulent time scale } \\ \phi_{s} & \text { Polar angle to diffracted ray } \\ \Psi & \text { Polar angle relative to incident ray } \\ \omega & \text { Angle to observer from } \\ & \text { upstream nozzle axis } \\ \text { Abbreviations } & \text { Frequency } \\ \text { BBSAN } & \\ \text { CFD } & \text { Broadband shock-associated noise } \\ \text { FUN3D } & \text { Computational fluid dynamics } \\ \text { LEE } & \text { Fully-unstructured Navier-Stokes } \\ \text { RANS } & \text { Linearized Euler equations } \\ \text { SPL } & \text { Reynolds-averaged Navier-Stokes } \\ \text { TTR } & \text { Sound pressure level } \\ & \text { Total temperature ratio } \\ & \end{array}$

*Research Aerospace Engineer, Aeroacoustics Branch, NASA Langley Research Center, 2 N. Dryden St. MS 461, Hampton, VA, 23681, USA, AIAA Senior Member, s.miller@nasa.gov 


\section{Introduction}

The fluid dynamics of heated, convecting, compressible turbulence that contains shock waves and PrandtlMeyer expansion waves is not well understood. Interactions between shock waves and turbulence creates shock-associated noise. One such canonical flow that produces shock-associated noise is the off-design supersonic jet. Off-design supersonic jets are observed in aerospace applications and include the exhaust of rockets and air-breathing engines (e.g. jet plumes). In practice, jet engines are integrated with the flight vehicle airframe, and this integration can impact the flow and noise. Broadband shock-associated noise (BBSAN), which is one component of jet noise, is scattered by the flight vehicle airframe. This paper presents a predominantly first principles approach, relative to others, using an acoustic analogy to predict the scattered BBSAN.

In this paper, we use the partially comprehensive acoustic analogy of Miller ${ }^{1}$ to predict the BBSAN component of jet noise in the presence of an airframe scattering surface. Model arguments are dependent on the vector Green's function of the linearized Euler equations (LEE), steady Reynolds-averaged NavierStokes (RANS) solutions, and the two-point cross-correlation of the equivalent source. The equivalent source is dependent on steady RANS solutions of the jet flow that capture the nozzle geometry and airframe surface. The jet mean flow and turbulent statistics are obtained by solving the steady RANS equations using a computational fluid dynamics (CFD) approach. Contours of the time-averaged streamwise velocity component and turbulent kinetic energy are examined with varying airframe position relative to the nozzle exit. Propagation effects are incorporated by approximating the vector Green's function of the LEE. This approximation involves the use of ray theory and an assumption that BBSAN is relatively unaffected by the refraction of the jet shear layer. The vector Green's function of the LEE is written as a function of the Green's function of the Helmholtz equation. The latter Green's function is calculated with the ray-tracing approach of Miller. ${ }^{2}$

This paper first surveys relevant measurements and prediction models for BBSAN, though no review of a prediction model for scattered BBSAN is presented, as this model is the first that includes scattering. The mathematical model, its associated arguments, and its implementation are described. Emphasis is placed on the physical meaning of the model arguments. Details of the approach to find the steady RANS solution are presented. A non-dimensional parameter is described to illustrate the deformation of the shock cells within the jet plume. A subset of the steady RANS solutions and BBSAN predictions are presented; the latter are compared with measurement. Finally, conclusions are drawn from the present theoretical investigation.

\section{A Survey of Previous Measurements}

A large number of investigators conducted careful measurements to study BBSAN. The first successful characterization of BBSAN was likely performed by Harper-Bourne and Fisher. ${ }^{3}$ They showed that BBSAN is caused by the interactions of coherent turbulence with the semi-periodic shock cell structure within the jet plume. BBSAN is dominant in intensity relative to other sources at mid- to high-frequencies in the upstream and sideline direction relative to the jet flow direction. The spectral content consists of multiple broad peaks that decrease in intensity with increasing frequency. Only a few years before the work of Harper-Bourne and Fisher, ${ }^{3} \mathrm{Yu}^{4}$ conducted near-field narrowband acoustic measurements from a wide range of jets. These measurements captured the near-field radiating nature of the noise intensity from off-design supersonic jets. Similar to the measurements of $\mathrm{Yu},{ }^{4}$ Tanna et al. ${ }^{5}$ conducted measurements using a convergent nozzle with $0.0508 \mathrm{~m}$ exit diameter and presented a large range of narrowband auto-spectra. The studies of $\mathrm{Yu}^{4}$ and Tanna et al. ${ }^{5}$ represented large scale measurement programs that made available large databases of acoustic measurements from off-design jets.

After the work of $\mathrm{Yu}^{4}$ and Tanna et al., ${ }^{5}$ the National Aeronautics and Space Administration, Langley Research Center, conducted a large number of measurements to study BBSAN. In particular, Seiner and Norum $^{6,7}$ conducted measurements to examine the shock-cell spacing with varying off-design parameter and correlated near-field microphone measurements with an intrusive hot-film probe. Comparisons were performed between on-design supersonic jets and equivalent off-design shock containing jets. The variation of overall sound pressure level (OASPL) between the on-design and off-design cases were quantified. Seiner and $\mathrm{Yu}^{8}$ conducted near-field correlations between microphones and also have shown correlations between early empirical prediction models. Their findings illustrated that the dominant noise source is located near the end of the potential core for the conditions they examined. Later, Seiner et al. ${ }^{9}$ examined BBSAN from rectangular nozzles. Norum and Seiner ${ }^{10,11}$ conducted measurements of static pressure, and near-field and 
far-field BBSAN auto-spectra, from unheated jets. The broadband component of shock-associated noise was isolated from the discrete component with the novel use of tabs on the nozzle lip.

Considerable effort was placed on quantifying the effect of forward flight on the BBSAN intensity and was an important component for system noise prediction methods. At NASA Langley, Norum and Shearin ${ }^{12}$ measured the variation of BBSAN in the far-field from under-expanded jets in the presence of flight vehicle Mach numbers, $M_{\infty} \leq 0.40$. They showed that measured BBSAN intensity varies little with varying $M_{\infty}$, and prediction techniques such as that devised by Harper-Bourne and Fisher ${ }^{3}$ were adequate. At higher flight vehicle Mach numbers, measurements of Ahuja et al. ${ }^{13}$ showed that there is more significant BBSAN intensity variation. Finally, Yamamoto et al. ${ }^{14}$ in their NASA contractor report, focused on producing shadowgraphs from six different nozzle configurations with varying jet operating conditions and $M_{\infty}$.

A number of contemporary measurements have been conducted. These measurements were mainly focused on the scaling of BBSAN intensity with stagnation temperature and on the separation of BBSAN from the total noise. Viswanathan et al. ${ }^{15}$ measured the saturation of BBSAN with stagnation temperature and separated the turbulent mixing noise from the BBSAN using incoherent spectral subtraction. Kuo et al. ${ }^{16}$ performed experimental studies to assess scaling laws of BBSAN intensity in the far-field with varying jet stagnation pressure and stagnation temperature. Similar to the earlier measurements of Seiner and $\mathrm{Yu},{ }^{8}$ Veltin et al. ${ }^{17}$ correlated various flow-field quantities with the acoustic field but used optical deflectometry. Panda ${ }^{18}$ showed that 'shock splitting' occurs within the jet, and is correlated with large organized vortices convecting over the shock train. Finally, similar to Norum and Seiner, ${ }^{10,11}$ Andre et al. ${ }^{19}$ investigated the effect of the discrete shock-associated tone on the broadband component.

\section{Previous Prediction Approaches and their Relation to the Present}

Many researchers created theoretical models for the prediction of BBSAN. Perhaps the first successful prediction method for BBSAN was created by Harper-Bourne and Fisher. ${ }^{3}$ The model was based on the premise of coherent interaction between turbulence in the jet shear layer and the nearly periodic jet shock cell structure. This interaction was modeled as a series of correlated point sources that combine constructively and destructively. Harper-Bourne and Fisher's ${ }^{3}$ model depended on the rate of decay of turbulence between shocks and a characteristic spectrum produced by each shock wave shear layer interaction. These quantities were obtained with least square fits to match the far-field data.

$\mathrm{Tam}^{20}$ developed a stochastic model for BBSAN where the basic physical model was described by Tam and Tanna. ${ }^{21}$ The shock cell structure was modeled as a waveguide following the method of Pack ${ }^{22}$ and the effect of divergence and turbulent dissipation was included following the method of Tam et al. ${ }^{23}$ The large-scale turbulence in the jet shear layer was modeled as a random superposition of instability waves supported by the jet mean flow, as described by Tam and Chen. ${ }^{24}$ Tam $^{25}$ modified the model of Tam ${ }^{20}$ to predict BBSAN from heated jets with a moderate off-design parameter. Tam and Reddy ${ }^{26}$ developed a method for the prediction of BBSAN from rectangular nozzles with aspect ratios up to six.

Morris and Miller ${ }^{27}$ developed a prediction method for BBSAN that is based upon an acoustic analogy approach. The Euler equations were rearranged into the LEE operator and were equated to equivalent sources. The convolution of the equivalent sources with the vector Green's function of the LEE resulted in a closed-form analytic expression for the spectral density of the pressure. Equivalent sources were modeled based upon a two-point cross-correlation and steady RANS solution. The two-point cross-correlation was modeled using dimensional arguments involving the shock pressure, turbulent velocity fluctuations, and local scales of turbulence. This formulation was based upon a separable model approach that is similar to the model of Ribner. ${ }^{28}$ The resultant model consisted of a volume integral containing the jet plume and an integral of the shock cell pressure wavenumber spectrum. Their acoustic analogy successfully predicted BBSAN for a wide range of single-stream jet operating conditions.

The model of Morris and Miller ${ }^{27}$ was extended by Miller and Morris ${ }^{29}$ to predict BBSAN from dualstream and rectangular jets. Their model made no assumptions regarding the geometry of the nozzle. The canonical BBSAN models of Harper-Bourne and Fisher, ${ }^{3}$ Tam, ${ }^{20}$ and Miller and Morris ${ }^{29}$ do not account for the refraction of sound through the jet shear layer. Miller and Morris ${ }^{30}$ studied propagation of BBSAN by altering the vector Green's function of the LEE to contain the Green's function of Lilley's equation as an argument. Henry et al. ${ }^{31}$ studied propagation of BBSAN by using a ray tracing approach. The Green's function of Lilley's equation within Miller and Morris $^{30}$ was found numerically using an adjoint approach and a locally parallel flow assumption. It was shown that in the upstream and sideline radiation direction refraction effects have little effect on BBSAN. In the downstream direction refraction has a larger effect but 
the source of BBSAN is relatively less intense. However, a small improvement in prediction accuracy was shown relative to predictions excluding refraction effects.

As jet stagnation temperature increases the resultant intensity of BBSAN increases then 'saturates' (ceases to increase with increasing temperature) as observed experimentally by Viswanathan et al. ${ }^{15}$ The model of Harper-Bourne and Fisher ${ }^{3}$ and Morris and Miller ${ }^{27}$ do not account for varying jet stagnation temperature while the model of $\mathrm{Tam}^{25}$ does. This latter model accounts for slight decreases of BBSAN intensity at all angles with increasing temperature using a simple empirical correction factor that is proportional to the inverse of the fully expanded density. Miller ${ }^{32}$ reformulated the model of Morris and Miller ${ }^{27}$ and included BBSAN refraction effects using the approach of Miller and Morris ${ }^{30}$ to account for BBSAN saturation with increasing temperature. Miller ${ }^{32}$ accurately predicted the saturation of BBSAN and proposed that saturation is due to an exact balance between increasing source strength and decreasing refraction intensity amplification.

Very recently, Miller ${ }^{1}$ created an acoustic analogy for jet noise that included equivalent sources for both turbulent mixing noise and BBSAN. It was shown to predict the total noise accurately for a wide range of jet Mach numbers and jet stagnation temperatures. The turbulent mixing noise and BBSAN were also predicted independently. The model made no assumptions regarding fine-scale or large-scale similarity spectra or sources, shear-noise or self-noise equivalent sources, or other fictitious sources.

A scattering approach for jet mixing noise was recently developed by Miller. ${ }^{2}$ They adopted the acoustic analogy of Miller ${ }^{33}$ that was created for the prediction of jet noise ground effects. The Green's function of Lilley's equation was approximated by creating a bridging function of asymptotic solutions. It contained arguments involving the locally parallel mean flow and the Green's function of the convective Helmholtz equation. A ray tracing solver, for the Green's function of the convective wave equation, was used to find this latter argument of the bridging function. Accurate predictions of jet mixing noise from jets operating over a wide range of Mach numbers and temperature ratios agreed with the measurements of Brown. ${ }^{34}$ Like the approach created for scattered jet mixing noise of Miller ${ }^{33}$ and Miller, ${ }^{2}$ the prediction method presented here is complimentary, but for scattered BBSAN.

\section{Mathematical Approach}

The predictions shown within this paper are based upon the partially comprehensive acoustic analogy of Miller. ${ }^{1}$ The far-field model equation contains equivalent source terms for the mixing and BBSAN components of jet noise. Details of the developed equation are shown in Miller ${ }^{1}$ and are heavily based upon the theories of Tam and Auriault, ${ }^{35}$ Morris and Farassat, ${ }^{36}$ and Morris and Miller. ${ }^{27}$ The model is dependent on steady RANS solutions and an analytical model of the two-point cross-correlation of the equivalent sources of mixing noise and BBSAN. The spectral density, $S$, at observer location, $\boldsymbol{x}$, is

$$
\begin{array}{r}
S(\boldsymbol{x}, \omega)=2 \pi^{3 / 2} \rho_{\infty}^{2} c_{\infty}^{6} \int_{-\infty}^{\infty} \int_{-\infty}^{\infty} \int_{-\infty}^{\infty} \frac{l_{x} l_{y} l_{z} x^{2} \tau_{s}}{c_{\infty}^{2} x^{2}+\left(\bar{u} x_{1}+\bar{v} x_{2}+\bar{w} x_{3}+c_{\infty} x\right)^{2} \tau_{s}^{2} \omega^{2}} \\
\times \exp \left[\frac{-\left(l_{x}^{2} x_{1}^{2}+l_{y}^{2} x_{2}^{2}+l_{z}^{2} x_{3}^{2}\right) \omega^{2}}{4 c_{\infty}^{2} x^{2}}\right]\left\{\pi_{g}^{* 0}(\boldsymbol{x} ; \boldsymbol{y}, \omega) \pi_{g}^{0}(\boldsymbol{x} ; \boldsymbol{y}, \omega) A_{s}^{2} \frac{\left(u_{s} / c_{\infty}\right)^{4}}{\tau_{s}^{2}}\right. \\
+\sum_{n=1}^{3} \sum_{m=1}^{3} \pi_{g}^{* n}(\boldsymbol{x} ; \boldsymbol{y}, \omega) \pi_{g}^{m}(\boldsymbol{x} ; \boldsymbol{y}, \omega)\left(B_{s}^{2} \frac{\left(u_{s} / c_{\infty}\right)^{2} u_{s}^{4}}{l_{x}^{2}}\right. \\
\left.\left.+C_{s}^{2} \frac{a_{m n} k p_{s}}{2 \pi \bar{\rho}^{2} \bar{u}^{2} l_{x}^{2}} \int_{-\infty}^{\infty} \tilde{p}_{s}\left(k_{1}, y_{2}, y_{3}\right) \exp \left[-i k_{1} y_{1}\right] d k_{1}\right)\right\} d \boldsymbol{y},
\end{array}
$$

where the integrand is a combination of spatially varying equivalent sources of mixing noise, BBSAN, and the vector Green's function of the LEE. The integral is restricted to the jet plume. The observer vector $\boldsymbol{x}$ is a vector from the nozzle exit to the observer, and the source vector $\boldsymbol{y}$ is a vector from the nozzle exit to the sources. Note that bold symbols denote vectors and a non-bold counterpart represents the magnitude of the vector. The angular frequency is $\omega=2 \pi f$, where $f$ is the frequency. Spatially varying time-averaged quantities consist of the velocity, $\overline{\boldsymbol{u}}=\overline{\boldsymbol{u}}(\bar{u}, \bar{v}, \bar{w})$, and density, $\bar{\rho}$. Statistical quantities related to the RANS closure are the turbulent kinetic energy, $k$, and specific dissipation, $\epsilon$. Turbulent scales, that are related to the RANS solution, are the streamwise, $l_{x}$, and cross-stream lengths scales, $l_{y}$ and $l_{z}$, the velocity $u_{s}$, and time scale, $\tau_{s}$. The coefficient matrix $a_{m n}$ describes anisotropic effects on the BBSAN component of 
the total noise and can potentially be described following the work of Khavaran. ${ }^{37}$ Here, we let $a_{m n}$ be unity. Ambient reference values are denoted by subscript $\infty$. The shock pressure, $p_{s}=p-p_{\infty}$, represents the pressure due to the shock cell structure. Within the inner integrand, the wavenumber spectrum of $p_{s}$ is denoted by $\tilde{p}_{s}$, and $k_{1}$ is the wavenumber.

Though Eqn. 1 is relatively complicated, individual terms have physical meaning. The first and second terms within the integrand are a result of analytical integration of a model for the two-point cross-correlation of the equivalent sources within the jet. For simplicity, it is assumed that this correlation has the same functional form for all equivalent sources. The first term within the integrand controls scaling of intensity for all sources with respect to the turbulent scales, $\overline{\boldsymbol{u}}$, and $\omega$. The second term within the integrand is a direct result of the mathematical form of the two-point cross-correlation, that (in this model) is assumed Gaussian for simplicity. Though, a better model would likely involve a combination of Gaussian and exponential decay with separation distance in temporal, radial, and axial directions, this approach only assumes the former. The first and second terms within the braces correspond to the mixing noise generated by fluid dilatation and unsteady forces per unit volume. Together they predict the total mixing noise produced by the jet. The last term within the braces of the integrand is the source term of BBSAN. BBSAN intensity is proportional to the turbulent kinetic energy, $k$, and the shock pressure, $p_{s}$, squared. The integral involving $k_{1}$ reduces to the shock pressure within the jet.

The turbulent scales are related to a steady RANS solution by, $l_{x}=c_{l} k^{3 / 2} \epsilon^{-1}, \tau_{s}=c_{\tau} k \epsilon^{-1}$, and $u_{s}=c_{u}(2 k / 3)^{1 / 2}$, where $c_{l}, c_{\tau}$, and $c_{u}$, are constant coefficients. These coefficients are calibrated with an over- and under-expanded jet at the sideline observer location. The under-expanded jet operates at $M_{j}=1.50$ and total temperature ratio, TTR $=1.00$, from a convergent nozzle with exit diameter of 0.0508 $\mathrm{m}$. The over-expanded jet operates at $M_{j}=1.29$ and TTR $=1$ from a $M_{d}=1.50$ nozzle with exit diameter of $0.0508 \mathrm{~m}$. Once the coefficients are obtained they are held constant regardless of nozzle geometry or jet operating condition. Also, we let $C_{s}$ equal unity. The coefficients are $c_{\tau}=1.25$ and $c_{l}=1.25$ for overexpanded flows and $c_{\tau}=0.50$ and $c_{l}=3.00$ for under-expanded flows. The cross-stream length scales are $1 / 3$ of the streamwise length scale. The convective velocity or Mach number is approximated as 7/10 the local value. Frequency dependence of the length scale has shown to improve predictions relative to measurement, especially for mixing noise, but demonstrates negligible improvement for BBSAN predictions. It could be included by following the approach of Lieb and Goldstein. ${ }^{38}$

Within Eqn. 1, $\pi_{g}^{n}$ represents the $n^{t h}$ or $m^{t h}$ logarithmic component of pressure. These components are governed by the solution of a set of partial differential equations

$$
\frac{\partial \pi_{g}^{n}}{\partial t}+\bar{v}_{j} \frac{\partial \pi_{g}^{n}}{\partial x_{j}}+\frac{\partial v_{g i}^{n}}{\partial x_{i}}=\delta(\boldsymbol{x}-\boldsymbol{y}) \delta(t-\tau) \delta_{0 n}
$$

and

$$
\frac{\partial v_{g i}^{n}}{\partial t}+\bar{v}_{j} \frac{\partial v_{g i}^{n}}{\partial x_{j}}+v_{g j}^{n} \frac{\partial \bar{v}_{i}}{\partial x_{j}}+\bar{c}^{2} \frac{\partial \pi_{g}^{n}}{\partial x_{i}}=\delta(\boldsymbol{x}-\boldsymbol{y}) \delta(t-\tau) \delta_{i n},
$$

where $v_{g i}^{n}$ represents the velocity component. The Dirac delta function is $\delta$ and $\delta_{i j}=1$ for $i=j$ and 0 for $i \neq j$. Assuming that the flow is statistically stationary, we transform $\pi_{g}^{n}$ from the time to frequency domain

$$
\pi_{g}^{n}(\boldsymbol{x} ; \boldsymbol{y}, \omega)=\frac{1}{2 \pi} \int_{-\infty}^{\infty} \pi_{g}^{n}(\boldsymbol{x}, \boldsymbol{y}, t-\tau) \exp [-i \omega(t-\tau)] d t
$$

where $\tau$ is the retarded time.

This mathematical survey is focused on BBSAN and not the total noise or mixing noise (see Miller ${ }^{1}$ for details), thus we focus on isolating the BBSAN component of the total noise from Eqn. 1. First, the coefficients within Eqn. 1, $A_{s}$ and $B_{s}$, are equated to zero and after simplification Eqn. 1 is

$$
\begin{array}{r}
S(\boldsymbol{x}, \omega)=\int_{-\infty}^{\infty} \int_{-\infty}^{\infty} \int_{-\infty}^{\infty} \frac{\pi^{1 / 2} C_{s}^{2} \rho_{\infty}^{2} c_{\infty}^{6} \pi_{g}^{* n} \pi_{g}^{m} k l_{x} l_{y} l_{z} p_{s} x^{2} \tau_{x}^{2}\left(c_{\infty}^{2} x^{2}+\left(\bar{u} x_{1}+\bar{v} x_{2}+\bar{w} x_{3}+c_{\infty} x\right)^{2} \tau_{s}^{2} \omega^{2}\right)}{} \\
\times \exp \left[-\left(l_{x}^{2} x_{1}^{2}+l_{y}^{2} x_{2}^{2}+l_{z}^{2} x_{3}^{2}\right) \omega^{2}\left(2 c_{\infty} x\right)^{-2}\right] \int_{-\infty}^{\infty} \tilde{p}_{s} \exp \left[-i k_{1} y_{1}\right] d k_{1} d \boldsymbol{y},
\end{array}
$$


where summations of $m$ and $n$ are implied from one to three and arguments of the terms are dropped for compactness. Equation 5 is evaluated by approximating the spatial integrals numerically. The majority of the arguments are dependent on the steady RANS solution in the vicinity of the airframe over the volume of the jet plume. The wavenumber of the shock pressure, $\tilde{p}_{s}$, is evaluated by performing a discrete Fourier transform in the direction of the flow. Integration of $\tilde{p}_{s} \exp \left[-i k_{1} y_{1}\right]$ is performed numerically (without the use of a Fourier transform library), and the limits of integration are restricted from $\left|k_{1}\right|>c$, where $c>0$ and represents a small constant. This approach removes the 'DC' component of the wavenumber frequency spectrum and has the advantage of retaining multiple dominant peaks of $\tilde{p}_{s}$. This approximate approach for evaluating the inner integral is beneficial because of the use of approximating the shock pressure, $p_{s}$, about $p_{\infty}$. A superior approach might use a shock-free base flow.

\section{The Scattered and Quiescent Field}

This paper compares the predicted BBSAN that is scattered by the airframe to the predicted BBSAN that propagates freely. This comparison requires that the steady RANS solution be evaluated both with and without the presence of the airframe. The presence of the airframe alters the statistics of the steady RANS solution, and these terms reside in the integrand of Eqn. 5. This approach captures the airframe's effect on the equivalent source.

The term, $\pi_{g}^{* n} \pi_{g}^{m}$, in Eqn. 5 alters the intensity due to propagation effects. Thus, a tailored form of $\pi_{g}^{n}$ captures the effect of the scattered field. Recall that jet shear layer refraction effects have little impact on the variation of far-field BBSAN auto-spectra. We adopt the approach of approximating the meanflow as either uniform or quiescent for propagation purposes only. In the absence of a mean flow (the source terms are still dependent on the steady RANS solution) Eqns. 2 and 3 are simplified, performing the Fourier transform with respect to time, and solving for $\pi_{g}^{n}$, results in the vector Green's function of the LEE for a quiescent environment

$$
\pi_{g}^{n}(\boldsymbol{x} ; \boldsymbol{y}, \omega)=-\frac{\delta_{i n}}{c_{\infty}^{2}} \int_{\boldsymbol{z}} \frac{\partial}{\partial z_{i}} \delta(\boldsymbol{z}-\boldsymbol{y}) g(\boldsymbol{x}, \boldsymbol{z}, \omega) d \boldsymbol{z}
$$

where $\boldsymbol{z}$ is a vector from source locations to observers. For free jet predictions

$$
g(\boldsymbol{x} ; \boldsymbol{z}, \omega)=\frac{\exp \left[i \omega|\boldsymbol{x}-\boldsymbol{z}| / c_{\infty}\right]}{4 \pi|\boldsymbol{x}-\boldsymbol{z}|} .
$$

Substituting Eqn. 7 into Eqn. 6 and simplifying yields an expression for the vector Green's function in the absence of varying mean flow (which is very similar to the Green's function of the Helmholtz equation)

$$
\pi_{g}^{n}(\boldsymbol{x} ; \boldsymbol{y}, \omega)=-\frac{i \omega x_{n}}{4 \pi c_{\infty}^{3} x^{2}} \exp \left[i \omega x / c_{\infty}\right] .
$$

When a scattering surface is placed in the field of the jet plume, a tailored form of $\pi_{g}^{n}$ must be found. We approximate the tailored form of $\pi_{g}^{n}$ by finding a tailored Green's function of $g$. Recall that BBSAN occurs at mid- to high-frequencies. Thus, one fast approach to calculate $g$ in the presence of a scattering surface is with the use of geometric acoustics. Recently, Miller ${ }^{2}$ showed that a ray tracing approach can be used to accurately predict jet mixing noise for mid- to high-frequencies. We use the same ray tracing approach for these predictions, and expect this approach to be a better approximation than previously, due to the fact that BBSAN occurs mainly at mid- to high-frequencies. The tailored Green's function is approximated as the sum of the incident and diffracted field

$$
g(\boldsymbol{x} ; \boldsymbol{y}, \omega)=g_{I}(\boldsymbol{x} ; \boldsymbol{y}, \omega)+\sum_{i=1}^{n} g_{D}(\boldsymbol{x} ; \boldsymbol{y}, \omega)
$$

where $g_{I}$ is the incident ray, equivalent to the free-field Green's function, and $g_{D}$ are the scattered rays. If the observer is in a shadow region, the incident ray is zero and the total diffracted field is the sum of all diffracted rays. Keller ${ }^{39}$ observed from Sommerfeld's ${ }^{40}$ solution of diffraction by a semi-infinite screen with a straight edge that incident waves propagating in a direction normal to the edge create a cylindrical diffracted wave centered on the edge. That is, when acoustic rays diffract they have similar properties to the initial incident rays but depend on the point of diffraction. Assuming that the amplitude of the diffracted ray is proportional to the amplitude at the point of diffraction, the acoustic pressure of the diffracted ray is 


$$
p(\boldsymbol{x}, \omega)=\tilde{D} p(\boldsymbol{E}, \omega)\left(\frac{r}{r_{1}\left(r_{1}+r\right)}\right)^{1 / 2} \exp \left[i k r_{1}\right],
$$

where $D$ is the diffraction coefficient and $\boldsymbol{r}_{1}=\boldsymbol{x}-\boldsymbol{E}$. The acoustic pressure field at the diffraction point on the edge is, $p(\boldsymbol{E}, \omega)=A \exp [i k \boldsymbol{E}]|\boldsymbol{E}-\boldsymbol{y}|^{-1}$, where $\boldsymbol{E}$ is the diffraction location on the edge and $A$ is a constant. Keller ${ }^{39}$ showed that Eqn. 10 satisfies Sommerfeld's ${ }^{40}$ exact asymptotic high-frequency solution for diffraction of a wave by a half-plane. After adopting a simplification from Agarwal et al. ${ }^{41}$ the resultant diffraction coefficient is

$$
\tilde{D}=\frac{\nu \exp [i \pi / 4]}{(2 \pi k)^{1 / 2}} \frac{\sin [\nu \pi]}{\sin \theta}\left[\frac{1}{\cos [\nu \pi]-\cos \left[\nu\left(\phi_{s}-\phi_{o}\right)\right]}+\frac{1}{\cos [\nu \pi]-\cos \left[\nu\left(\phi_{s}+\phi_{o}+\pi\right)\right]}\right],
$$

where $\phi_{s}$ is the polar angle to the incident ray, $\phi_{o}$ is the polar angle to the diffracted ray, $\theta$ is the oblique angle between the edge and incident ray, $\nu=\pi / \beta$, and $\beta$ is the wedge angle. The contribution to $g_{D}$ from a single diffracted ray is calculated using Eqns. 10 and 11. All diffracted ray contributions are used to compute the tailored Green's function, $g$.

\section{Quantification with a Non-Dimensional Number}

We now turn our attention to the development of a non-dimensional number that quantifies the effect of the airframe on the BBSAN source. Parameters are identified that alter the noise source with varying jet operating condition and airframe position relative to the nozzle exit.

We propose a non-dimensional number $\Gamma$

$$
\Gamma=\frac{D_{j} x_{p} \tan \left[\delta_{\eta}\right]}{y_{p}\left(y_{p}-D / 2\right)}
$$

where $D$ is the nozzle diameter, $D_{j}$ is the fully expanded diameter, $x_{p}$ and $y_{p}$ are axial and radial vector components that form a vector from the nozzle exit to the trailing edge, and $\delta_{\eta}$ is the spreading rate of the jet. Physically, Eqn. 12 is the ratio of the product of jet and airframe length scales divided by the cross-stream length scale and interaction distance. Small values of $\Gamma$ imply that airframe effects on the BBSAN sources are negligible. Likewise, large values of $\Gamma$ imply that the effects of the airframe on the BBSAN sources are very large. Note that $y_{p}>D / 2$.

The fully expanded diameter is

$$
D_{j}=D\left(\frac{1+\frac{\gamma-1}{2} M_{j}^{2}}{1+\frac{\gamma-1}{2} M_{d}^{2}}\right)^{(\gamma+1) / 4(\gamma-1)}\left(\frac{M_{d}}{M_{j}}\right)^{1 / 2},
$$

where $\gamma$ is the ratio of specific heats. Values of $\delta_{\eta}$ are not readily available without numerical calculations or measurement. The spreading rate is used to estimate the jet impingement location (used in the development of Eqn. 12). The jet impingement location is estimated as

$$
x_{I}=\frac{y_{p}-D / 2}{\tan \left[\delta_{\eta}\right]} .
$$

Additional details regarding the development and significance of $\Gamma$ are discussed by Miller. ${ }^{2}$

\section{Results}

The prediction method outlined in the previous section is validated with a series of measurements conducted by Brown. ${ }^{34}$ These measurements were performed with the Small Hot Jet Acoustic Rig (SHJAR) at NASA using the small metal chevron (SMC) series of nozzles. Large flat plates, described by Brown, ${ }^{34}$ were placed near various SMC nozzles. A photograph of the experiment is shown in Fig. 1 courtesy of Podboy. ${ }^{42}$ The nozzle and large flat plate can be seen on the right side of the photograph. The nozzle is pointed towards the camera and the large flat plate is mounted vertically. An array of microphones is shown on stands centered about the nozzle exit. Note that the plate is between the nozzle exit and the microphone array. 
The experiment of Brown ${ }^{34}$ is summarized in the diagram of Fig. 2. The axial distance from the nozzle exit to the trailing edge of the plate is, $x_{p}$, and the radial distance from the nozzle centerline to the plate is, $y_{p}$. These two quantities and the jet operating condition were varied by Brown ${ }^{34}$ to study the scattered jet noise. In the measurements the polar microphone array distance was $R / D=75$, and the results have been scaled to $R / D=100$ for comparison with predictions. Additional corrections were made to account for atmospheric absorption. The observer angle, $\Psi$, is measured from the jet upstream axis. For full details of the measurement program see Bridges and Brown, ${ }^{43}$ Podboy ${ }^{42}$ and Brown. ${ }^{34}$

\section{Steady Reynolds-Averaged Navier-Stokes Solutions}

The steady RANS solutions are calculated numerically using the fully-unstructured Navier-Stokes (FUN3D) solver developed at NASA Langley Research Center. For more information on FUN3D see Anderson and Bonhaus ${ }^{44}$ or Nielsen. ${ }^{45}$ We focus on the SMC016 nozzle, which is a convergent-divergent nozzle with design Mach number, $M_{d}=1.50$, and exit diameter, $D=0.0508 \mathrm{~m}$. The computational domain extends $100 D$ downstream from the nozzle exit, $50 D$ in the radial direction from the centerline, and $5 D$ upstream from the nozzle inlet. Numerical solutions are governed by the steady RANS equations closed by the Menter shear stress transport $(\mathrm{SST})^{46}$ turbulence model. Roe ${ }^{47}$ flux vector construction is used for spatial discretization and is second order accurate. A Roe ${ }^{48}$ 'modmin' flux limiter is used in cases that fail to converge due to very large fluxes in the initial transient solution. The flow-field is initialized as quiescent at the start of the simulation. The validation of the FUN3D steady RANS solutions for single-stream jets was performed in an earlier study by Smith and Miller. ${ }^{49}$

One steady RANS solution is shown in Fig. 3 for a jet operating at $M_{j}=1.29$ and TTR $=1$. The nozzle is convergent-divergent, designed with the method of characteristics, with design Mach number, $M_{d}=1.50$ and $D=0.0508 \mathrm{~m}$. The computational domain contains the nozzle geometry and a large flat plate located at $x_{p} / D=10$ and $y_{p} / D=-2$. Reference Fig. 2 for the definition of $x_{p}$ and $y_{p}$. For these jet conditions and plate position the non-dimensional number, $\Gamma=0.309$, signifies that the BBSAN source is deformed relatively little. Note that the simulations are fully three-dimensional, and these figures only illustrate a plane through the nozzle centerline axis and normal to the plate surface. Figure 3(a) shows the unstructured-structured computational domain in the $x-y$ plane. The jet plume has been discretized using a cartesian grid and the exterior region has been discretized using an unstructured grid. The $x$-axis and $y$-axis have been normalized by $D$. The nozzle contour, with nozzle exit located at the coordinate system origin, and flat plate terminating at $x_{p} / D=10$ and $y_{p} / D=-2$, are shown in each sub-figure. There are ample grid points to resolve the shock-cell structure within the jet plume, and the boundary layer is resolved along the plate surface facing the jet flow.

Figures 3(b), 3(c), and 3(d) show contours of $\bar{\rho}, \bar{u}$, and $k$, respectively. In this particular case, it is clear that the entire shock-cell structure is shielded by the airframe surface relative to observers in the sideline and upstream direction. The field-variables, $\bar{\rho}$ and $\bar{u}$, shown in Figs. 3(b) and 3(c), have little deformation due to the airframe surface. Note that the contour lines relative darkness corresponds to the subfigure legend. That is, they are nearly symmetric about the $x-z$ plane. This is in contrast with the turbulent kinetic energy in Fig. 3(d), that does show significant asymmetry, especially so relative to $\bar{\rho}$ and $\bar{u}$. Here, the relatively weak deformation of the acoustic source intensity and location caused by the presence of the airframe are captured by the steady RANS solution.

We now examine the effect of the position of the airframe surface relative to the $M_{j}=1.29$ and TTR $=1$ jet. Figure 4 shows contours of $\bar{u}$ for $x_{p}=4$ and $y_{p} / D=-6,-4,-2$, and -1 . Values of $\Gamma$ are 0.011 , 0.027, 0.124, and 0.741 for Figs. 4(a), 4(b), 4(c), and 4(d), respectively. Contours within Figs. 4(a) and 4(b) are nearly identical, while in Figs. 4(c) and 4(d), the potential core length has slightly increased. Though, it appears that the flow is still relatively symmetric about the $x-z$ plane. Miller ${ }^{2}$ showed that as the jet approaches the surface, the entrainment is affected between the jet flow and the airframe, and there is additional entrainment acceleration compared to the unshielded side of the jet.

Figures 5(a), 5(b), 5(c), and 5(d) show contours of $k$ for the same jet condition and airframe position as Fig. 4. Values of $\Gamma$ are the same. Contours of $k$ are very similar between $y_{p} / D=-4$ and -6 , and change little when examining steady RANS solutions calculated farther from the surface. Unlike the steady contours of $\bar{u}$, significant asymmetry is observed at $y_{p} / D=-2$ and -1 , in Figs. 5(c) and 5(d). The amount of production of $k$ between the jet and airframe, for $y_{p} / D=-2$, is significantly decreased while the contours of $k$ on the opposite side are very similar to the undisturbed cases. This is due to the decreased relative shear layer velocity between the jet and airframe as observed in Fig. 4(c). Figure 5(d) shows an opposite trend relative 
to Fig. 5(c), as there are additional levels of $k$ between the jet and airframe. The additional levels are due to the direct interaction of part of the jet plume with the airframe surface.

We now examine the steady RANS solutions of $\bar{u}$ and $k$ for the same conditions but extend the airframe surface to $x_{p} / D=10$. The results are shown in Fig. 6 for $\bar{u}$ and Fig. 7 for $k$. Values of $\Gamma$ for $y_{p} / D=-6$ through $y_{p} / D=-1$ are $0.028,0.066,0.309$, and 1.853 respectively. Variation of $\bar{u}$ in Fig. 6 is very small except for $y_{p} / D=-1$, as shown in Fig. 6(d), where the jet shows a very weak Coanda like effect. This can likely be attributed to the additional plane-form of the plate relative to the $x_{p} / D=-4$ case. Variation of $k$ with $y_{p} / D$ is shown in Fig. 7 , and at $y_{p} / D=-4$ and -6 little difference is observed. Larger asymmetries about the $x-z$ plane are observed in Figs. $7(\mathrm{c})$ and $7(\mathrm{~d})$. These changes in $k$ are much larger than the $x_{p} / D=4$ case shown previously in Fig. 5. The magnitude of $k$ in the region of $y / D<0$ is lower than $y / D>0$. The BBSAN source is modified as it scales with $k$, especially so on the shielded side of the jet flow. Larger values of $\Gamma$ that are approximately unity or greater indicate this modification.

A final contour set of $\bar{u}$ and $k$ are shown in Figs. 8 and 9 respectively. The plate is extended to $x_{p} / D=20$ and the other conditions remain the same. Values of $\Gamma$ for $y_{p} / D=-6$ through $y_{p} / D=-1$ are $0.056,0.132$, 0.618 , and 3.706 respectively. These predictions are very similar to those presented in Figs. 6 and 7 . We observe the same Coanda effect (an attachment of the jet flow upon the airframe surface) in Fig. 8(d) as we did in Fig. 6(d). The extension of the plate by an additional $10 D$ has caused little discernible difference in $\bar{u}$ for this supersonic jet flow. There are some differences in the contours of $k$ between $x_{p} / D=10$ and 20 , but the contours follow the same trend. In particular, the peak $k$ in the shear layer between the jet and airframe has moved closer to the nozzle exit and decreased in magnitude. Generally, the deformation of contours of $\bar{u}$ are relatively small, even for plate positions of $y_{p} / D=-1$. Contours of $k$ are much more sensitive to the position of the plate, and the importance of intensity of $k$ is shown in the equivalent source model, where the BBSAN intensity is proportional to $k$. The deformation of $k$ for the cases examined is correlated with $\Gamma$.

\section{Broadband Shock-Associated Noise Predictions}

Here, we will present predictions of scattered and isolated BBSAN. Figure 10 shows predictions compared with measurements of acoustic spectra produced by a $M_{j}=1.29$ and TTR $=1$ off-design jet. The design Mach number is 1.50 and the exit diameter is $0.0508 \mathrm{~m}$. The $y$-axis represents sound pressure level (SPL) per unit $S t$, where $S t$ is the Strouhal number, $S t=f D_{j} u_{j}^{-1}$. The observer angle, $\Psi$, is shown in the upper right corner of each figure and observers are $100 \mathrm{D}$ from the nozzle exit. The plate edge is located at $x_{p} / D=4$ and $y_{p} / D=-2$. The solid and dashed lines represent the free (Bridges and Brown ${ }^{43}$ ) and scattered $\left(\right.$ Brown $\left.^{34}\right)$ jet measurements, respectively. Lines with circles and triangles represent predictions for free turbulent mixing noise and BBSAN, respectively, using the method of Miller. ${ }^{1}$ Predicted scattered jet mixing noise is represented by dashed lines with diamonds and was performed by Miller. ${ }^{2}$ Predictions of jet surface interaction noise of Miller ${ }^{50}$ are shown as a line with squares. These predictions are shown to illustrate the different components of the total jet noise for the scattered and isolated jet configurations. Some of these components have been isolated experimentally using the approach of Viswanathan, ${ }^{51}$ and are similar to present predictions. A description of the physics of these components relative to the presented predictions and measurements is outside the scope of this paper. The focus of this paper is scattered BBSAN as predicted by Eqn. 5 that is represented by dashed lines with grads.

Within Fig. 10, except at $\Psi^{o}=50$, there is very little change in the measured isolated and scattered BBSAN. The predictions show larger amounts of shielding relative to measurement at higher frequencies. The relative intensity at the peak frequency of BBSAN is captured accurately, as can be seen at $\Psi^{o}=50$. Also, the peak frequency of the scattered BBSAN is higher than the isolated case in this direction. Here, $\Gamma=0.124$ and the steady RANS flow-field is shown in Figs. 4(c) and 5(c). This relatively small value of $\Gamma$ signifies that the BBSAN source is relatively unaffected by the airframe, and the relative differences between the scattered and isolated BBSAN predictions are mainly due to propagation effects.

Figures 11 through 14 show predictions and measurements produced by the $M_{j}=1.29$ and TTR $=1$ offdesign jet, but with varying $y_{p} / D$ locations. The values of $y_{p} / D$ are $-4,-6,-8$, and -10 , with corresponding values of $\Gamma=0.027,0.011,0.006$, and 0.004 . Time-averaged steady RANS solutions for these cases are shown in Fig. 4 for $\bar{u}$ and Fig. 5 for $k$. As with the $y_{p} / D=-2$ case, these values are relatively small and the variation in BBSAN between the scattered and isolated case is due to propagation. At $\Psi^{o}=90$ and 110, no measured difference is observed between the scattered and isolated BBSAN. This is likely due to the fact that the dominant sound producing shock wave shear layer interactions are not shielded relative to the observer. Recall that the dominant source is generally near the end of the potential core, and this region is 
not shielded by the airframe surface. Prediction and measurement for the scattered and isolated BBSAN are sometimes identical, especially when the observer is not shielded and $y_{p} / D$ is large, which gives credibility to the developed approach. It is observed that BBSAN is increasingly shielded and its peak frequency increases while $\Psi^{o}=50$ and $x_{p} / D=4$ and the magnitude of $y_{p} / D$ increase. Predictions capture this trend observed in intensity and frequency. By holding $\Psi^{o}=70$ and $x_{p} / D=4$ constant while increasing $y_{p} / D$, we initially observe that the peak magnitudes of scattered BBSAN and isolated BBSAN are in general agreement in prediction and measurement. At $y_{p} / D=-6$, a slight change occurs, and then for $y_{p} / D \geq-8$, a large reduction of scattered BBSAN is observed. Predictions capture this 'delayed' reduction of scattered BBSAN intensity between $y_{p} / D=-6$ and $y_{p} / D \leq-8$.

For the next case, the same jet operating conditions are retained, $M_{j}=1.29$ and $\mathrm{TTR}=1$, and the plate is extended to $x_{p} / D=10$. Predictions and corresponding measurements are shown in Figs. 15 through 18. The values of $y_{p} / D$ are $-2,-4,-6$, and -10 , respectively. Values of $\Gamma$ are $0.309,0.066,0.028$, and 0.010 . Time-averaged steady RANS solutions for these cases are shown in Fig. 6 for $\bar{u}$ and Fig. 7 for $k$. Unlike the predictions shown for $x_{p} / D=4$, these predictions and measurements demonstrate significant shockassociated noise shielding at all angles examined. This is purely due to the extension of the plate by an additional $6 D$ and resulting propagation effects. The jet core length is approximately $8 D$ and the dominant BBSAN source oscillates in the region between approximately $6 D$ and $9 D$. By examining Fig. 6 , for example, for the observer angles chosen it can be observed that the propagation path is restricted to diffracting waves, and all predictions and measurements are shielded. The relatively small values of $\Gamma$ imply that the source of BBSAN is relatively unaffected by the airframe surface, and at most mildly for $y_{p} / D=-2$ and $\Gamma=0.309$. Very little difference in relative BBSAN shielding is observed between $y_{p} / D=-2$ and $y_{p} / D=-4$, even though there is a relatively more significant change in $\Gamma$, as shown in Figs. 15 and 16 . The quality of the scattered BBSAN predictions vary. Generally, higher frequencies are under-predicted, thus corresponding $\Delta \mathrm{dB}$ would be over-predicted. Though, this would be less of a problem for full-scale engines, because they can exhibit greater BBSAN intensity decay at high frequencies.

The final set of comparisons for the $M_{j}=1.29$ and TTR $=1$ jet are shown for $x_{p} / D=20$, and all other conditions are held constant. Predictions and corresponding measurements are presented in Figs. 19 through 21. The values of $y_{p} / D$ are $-2,-6$, and -10 , respectively. Values of $\Gamma$ are $0.618,0.056$, and 0.020 for $y_{p} / D=-2,-6$, and -10 . Time-averaged steady RANS solutions for these cases are shown in Fig. 8 for $\bar{u}$ and Fig. 9 for $k$. Like the predictions and measurements for $x_{p} / D=10$, these predictions also show significant airframe shielding at all observer angles and values of $y_{p} / D$. The scattered BBSAN predictions are more aligned with measurement than previous cases. This is likely due to the ray paths having relatively small angles relative to the airframe surface. This increases the accuracy of the ray tracing approach, as shown by Miller. ${ }^{2}$ For this reason, near $S t \approx 10$, predictions are in better agreement relative to previous cases, such as $x_{p} / D=4$. Though, in the downstream direction, peak scattered BBSAN frequencies are over-predicted relative to measurement by $S t \approx 2$. For the $y_{p} / D=-6$ and -10 cases, shown in Figs. 20 and 21 respectively, values of $\Gamma$ are very small, and relative error in the predictions and measurement of isolated and scattered BBSAN are purely due to propagation. Relative errors between scattered and isolated BBSAN predictions and measurement, in Fig. 19 where $y_{p} / D=-2$, are due to a combination of the ray tracing calculation and a slightly deformed BBSAN source. Though, the deformation of the BBSAN source by the airframe surface is captured through the steady RANS solution shown in Figs. 8(c) and 9(c). This analysis is complicated by the varying intensity of scattered jet mixing noise with varying $y_{p} / D$, that accounts for some of the total measured intensity, relative to the predicted scattered BBSAN intensity.

Perhaps one of the most interesting features of scattered BBSAN (that was not apparent in the initial development of the theory), are elegant 'double broad lobes,' that occur in certain circumstances. Recall that isolated BBSAN is characterized by multiple broad lobes, that decrease in intensity and spectral width with increasing frequency, that form due to constructive interference. Miller ${ }^{52}$ isolated these individual contributions to the total isolated BBSAN, in the context of the model of Morris and Miller ${ }^{27}$ and Tam. ${ }^{20}$ Scattered BBSAN, varying with frequency, is characterized by additional broad lobes at low frequencies, that increase in intensity and spectral width below the peak frequency, then decrease in intensity and spectral width above the peak frequency. Some examples of this unique scattered BBSAN spectrum can be observed in Fig. $13\left(x_{p} / D=4, y_{p} / D=-8\right.$, and $\Psi^{o}=70$ and 90$)$, Fig. $16\left(x_{p} / D=10, y_{p} / D=-4\right.$, and $\Psi^{o}=90$ and 110$)$, or Fig. $21\left(x_{p} / D=20, y_{p} / D=-10\right.$, and $\left.\Psi^{o}=110\right)$. Spectral lobes below the peak scattered BBSAN frequency are due to scattering effects. In a mathematical context, they are due to the presence of Fresnel integrals that are implicitly contained in analytical solutions of point sources 
about semi-infinite flat plates. Spectral lobes at and above the peak frequency are mainly due to traditional BBSAN constructive interference and partially due to scattering effects. Above the peak scattered BBSAN frequency, the combination of traditional constructive interference and interference due to scattering can have a strengthening or smoothing effect on the broadband noise. Nonetheless, the double broad lobes are an interesting phenomenon that is unique to shock-associated noise.

\section{Conclusion}

A mathematical model is developed for the prediction of scattered BBSAN and is based on the partially comprehensive acoustic analogy. Predictions using the model, for the scattered and isolated BBSAN, are compared with a wide variety of measurements involving a large flat plate. These predictions are generally in satisfactory agreement with measurement. The model arguments are dependent on the vector Green's function of the LEE, steady RANS solutions, and a two-point cross-correlation of the equivalent source.

The equivalent source is dependent on steady RANS solutions of the jet flow that contain the effects of the nozzle geometry and airframe surface. Steady RANS solutions are calculated for an off-design jet with various plate locations. Contours of the time-averaged streamwise velocity component and turbulent kinetic energy are examined with varying plate position. Generally, the turbulent statistics are more sensitive to the airframe than density, velocity, and pressure components. This is due to the sensitivity of turbulent kinetic energy on the shear layer gradient that changes due to the variation of entrainment velocity between the jet and airframe.

Propagation effects are captured by finding an approximation of the vector Green's function of the LEE. This approximation involves the use of ray theory and an assumption that BBSAN is relatively unaffected by the refraction of the jet shear layer. Also, the dominant energy in scattered BBSAN spectra are at relatively mid- to high-frequencies, and the ray theory assumption is accurate in these frequency bands.

Predictions of scattered and isolated BBSAN agree with measurements of equivalent scattered and isolated BBSAN. Generally, scattered BBSAN exhibits slightly increased peak frequencies. Also, shielded BBSAN exhibits greatly decreased intensities, and generally the model captures these peak intensity and peak frequency trends. The model under-predicts very high frequency scattered BBSAN, but the additional intensity from scattered turbulent mixing noise will increase the total intensity.

A non-dimensional parameter, $\Gamma$, is presented that quantifies the changes of the BBSAN source with varying jet operating conditions and airframe position. The steady RANS solutions, in particular the variation of turbulent kinetic energy with plate position, demonstrate a correlation between $\Gamma$ and turbulent kinetic energy. Acoustic predictions corresponding to a wide range of $\Gamma$, demonstrate that the model can predict the scattering of BBSAN, even with sources altered by the airframe. That is, the model predicts scattered BBSAN when the source changes due to the presence of an airframe. It is more likely that the approximation of the vector Green's function of the LEE with the use of ray theory causes more error in prediction than the choice of the equivalent source of BBSAN.

Most interestingly, double broad lobes are observed in the predicted scattered BBSAN. Below the peak frequency of BBSAN the lobes are due to diffraction, and above the peak frequency the lobes are due to traditional constructive interference. The lobes decrease in intensity as the norm of frequency increases relative to the peak frequency. It is likely that the traditional constructive interference, that occurs at high frequencies, is disturbed by the constructive contribution due to diffraction.

\section{Acknowledgments}

The author is grateful for continuous support from the National Aeronautics and Space Administration Fundamental Aeronautics Program High Speed Project. Matthew J. Smith provided the steady Reynoldsaveraged Navier-Stokes solutions. 


\section{References}

${ }^{1}$ Miller, S. A. E., "Toward a Comprehensive Model of Jet Noise using an Acoustic Analogy," AIAA Journal, Vol. 52, No. 10, 2014, pp. 2143-2164. doi:10.2514/1.J052809.

${ }^{2}$ Miller, S. A. E., "The Prediction and Analysis of Jet Flows and Scattered Turbulent Mixing Noise about Flight Vehicle Airframes," NASA/TM-2014-218506, 2014.

${ }^{3}$ Harper-Bourne, M. and Fisher, M. J., "The Noise from Shock-Waves in Supersonic Jets," AGARD Conference Proceedings, 1973.

${ }^{4} \mathrm{Yu}$, J. C., "Investigation of the Noise Field of Supersonic Axisymmetric Jet Flow," Ph.D. Dissertation, Syracuse University, 1971.

${ }^{5}$ Tanna, H. K., Dean, P. D., and Burrin, R. H., "The Generation and Radiation of Supersonic Jet Noise. Volume IV. Shock-Associated Noise Data," AFAPL-TR-76-65, 1976.

${ }^{6}$ Seiner, J. and Norum, T., "Experiments of Shock Associated Noise of Supersonic Jets," 12th Fluid and Plasma Dynamics Conference, AIAA Paper 79-1526, 1979. doi:10.2514/6.1979-1526.

${ }^{7}$ Seiner, J. and Norum, T., "Aerodynamic Aspects of Shock Containing Jet Plumes," 6th Aeroacoustics Conference, AIAA Paper 80-0965, 1980. doi:10.2514/6.1980-965.

${ }^{8}$ Seiner, J. and Yu, J. C., "Acoustic Near-Field Properties Associated with Broadband Shock Noise," AIAA Journal, Vol. 22, No. 9, 1984, pp. 1207-1215. doi:10.2514/3.8762.

${ }^{9}$ Seiner, J., Manning, J., and Ponton, M., "Acoustic Properties Associated with Rectangular Geometry Supersonic Nozzles," 10th Aeroacoustics Conference, AIAA Paper 86-1867, 1986. doi:10.2514/6.1986-1867.

${ }^{10}$ Norum, T. D. and Seiner, J. M., "Broadband Shock Noise from Supersonic Jets," AIAA Journal, Vol. 20, No. 12, 1982, pp. 68-73. doi:10.2514/3.51048.

${ }^{11}$ Norum, T. D. and Seiner, J. M., "Measurements of Mean Static Pressure and Far Field Acoustics of Shock Containing Supersonic Jets," NASA-TM-84521, 1982.

${ }^{12}$ Norum, T. D. and Shearin, J. G., "Shock Noise from Supersonic Jets in Simulated Flight to Mach 0.4," 10th AIAA/CEAS Aeroacoustics Conference, AIAA Paper 86-1945, 1986. doi:10.2514/6.1986-1945.

${ }^{13}$ Ahuja, K. K., Tanna, H. K., and Tester, B. J., "Effects of Simulated Forward Flight on Jet Noise, Shock Noise, and Internal Noise," 5th AIAA Aeroacoustics Conference, Seattle, Washington, 12 - 14 March, AIAA Paper 79-0615, 1979. doi:10.2514/6.1979-615.

${ }^{14}$ Yamamoto, K., Brausch, J. F., Janardan, B. A., Hoerst, D. J., Price, A. O., and Knott, P. R., "Experimental Investigation of Shock-Cell Noise Reduction for Single-Stream Nozzles in Simulated Flight, Comprehensive Data Report. Volume 3: Shadowgraph Photos and Facility Description," NASA CR-168234, 1984.

${ }^{15}$ Viswanathan, K., Alkislar, M. B., and Czech, M. J., "Characteristics of the Shock Noise Component of Jet Noise," AIAA Journal, Vol. 48, No. 1, 2010, pp. 25-46. doi:10.2514/1.38521.

${ }^{16}$ Kuo, C., McLaughlin, D. K., and Morris, P. J., "Effects of Supersonic Jet Conditions on Broadband Shock-Associated Noise," 49th AIAA Aerospace Sciences Meeting, AIAA Paper 2011-1032, 2011. doi:10.2514/6.2011-1032.

${ }^{17}$ Veltin, J., Day, B. J., and McLaughlin, D. K., "Correlation of Flowfield and Acoustic field Measurements in High-Speed Jets," AIAA Journal, Vol. 49, No. 1, 2011, pp. 150-163. doi:10.2514/1.J050583.

${ }^{18}$ Panda, J., "Shock Oscillation in Underexpanded Screeching Jets," Journal of Fluid Mechanics, Vol. 363, 1998, pp. 173198. doi:10.1017/S0022112098008842.

${ }^{19}$ Andre, B., Castelain, T., and Bailly, C., "Broadband Shock-Associated Noise in Screeching and Non-Screeching Underexpanded Supersonic Jets," AIAA Journal, Vol. 51, No. 3, 2013, pp. 665-673. doi:10.2514/1.J052058.

${ }^{20}$ Tam, C. K. W., "Stochastic Model Theory of Broadband Shock-Associated Noise from Supersonic Jets," Journal of Sound and Vibration, Vol. 116, No. 2, 1987, pp. 265-302. doi:10.1016/S0022-460X(87)81303-2.

${ }^{21}$ Tam, C. K. W. and Tanna, H. K., "Shock-Associated Noise of Supersonic Jets from Convergent-Divergent Nozzles," Journal of Sound and Vibration, Vol. 81, No. 3, 1982, pp. 337-358. doi:10.1016/0022-460X(82)90244-9.

${ }^{22}$ Pack, D. C., "A Note on Prandtls Formula for the Wavelength of a Supersonic Gas Jet," Quarterly Journal of Applied Mathematics and Mechanics, Vol. 3, 1950, pp. 173-181.

${ }^{23}$ Tam, C. K. W., Jackson, J. A., and Seiner, J. M., "A Multiple-Scales Model of the Shock-Cell Structure of Imperfectly Expanded Supersonic Jets," Journal of Fluid Mechanics, Vol. 153, 1985, pp. 123-149. doi:10.1017/S0022112085001173.

${ }^{24}$ Tam, C. K. W. and Chen, K. C., "A Statistical Model of Turbulence in Two-Dimensional Mixing Layers," Journal of Fluid Mechanics, Vol. 92, No. 2, 1979, pp. 303-326. doi:10.1017/S002211207900063X.

${ }^{25}$ Tam, C. K. W., "Broadband Shock-Associated Noise of Moderately Imperfectly-Expanded Supersonic Jets," Journal of Sound and Vibration, Vol. 140, No. 1, 1990, pp. 55-71. doi:10.1016/0022-460X(90)90906-G.

${ }^{26}$ Tam, C. K. W. and Reddy, N., "Prediction Method for Broadband Shock-Associated Noise from Supersonic Rectangular Jets," Journal of Aircraft, Vol. 33, No. 2, 1996, pp. 298-303. doi:10.2514/3.46937.

${ }^{27}$ Morris, P. J. and Miller, S. A. E., "Prediction of Broadband Shock-Associated Noise Using Reynolds-Averaged NavierStokes Computational Fluid Dynamics," AIAA Journal, Vol. 48, No. 12, 2010, pp. 2931-2944. doi:10.2514/1.J050560.

${ }^{28}$ Ribner, H. S., "The Generation of Sound by Turbulent Jets," Advances in Applied Mechanics, Vol. 8, 1964, pp. 104-182.

${ }^{29}$ Miller, S. A. E. and Morris, P. J., "The Prediction of Broadband Shock-Associated Noise from Dualstream and Rectangular Jets Using RANS CFD," 16th AIAA/CEAS Aeroacoustics Conference, AIAA Paper 2010-3730, 2010. doi:10.2514/6.20103730 .

${ }^{30}$ Miller, S. A. E. and Morris, P. J., "The Prediction of Broadband Shock-Associated Noise Including Propagation Effects," International Journal of Aeroacoustics, Vol. 11, No. 8, 2012, pp. 755-782. doi:10.1260/1475-472X.11.7-8.755.

${ }^{31}$ Henry, C., Bailly, C., and Bodard, G., "Statistical Modeling of BBSAN Including Refraction Effects," 18th AIAA/CEAS Aeroacoustics Conference, AIAA Paper 2012-2163, 2012. doi:10.2514/6.2012-2163. 
${ }^{32}$ Miller, S. A. E., "The Scaling of Broadband Shock-Associated Noise with Increasing Temperature," 33rd AIAA Aeroacoustics Conference, AIAA Paper 2012-2215, 2012. doi:10.2514/6.2012-2215.

${ }^{33}$ Miller, S. A. E., "The Prediction of Jet Noise Ground Effects using an Acoustic Analogy and a Tailored Green's Function," Journal of Sound and Vibration, Vol. 333, No. 4, 2014, pp. 1193-1207. doi:10.1016/j.jsv.2013.10.028.

${ }^{34}$ Brown, C., "Jet-Surface Interaction Test: Far-Field Noise Results," J. Eng. Gas Turbines Power, Vol. 135, No. 7, 2011, pp. 7. doi:10.1115/1.4023605.

${ }^{35}$ Tam, C. K. W. and Auriault, L., "Jet Mixing Noise from Fine-Scale Turbulence," AIAA Journal, Vol. 37, No. 2, 1999, pp. 145-153. doi:10.2514/2.691.

${ }^{36}$ Morris, P. J. and Farassat, F., "Acoustic Analogy and Alternative Theories for Jet Noise Prediction," AIAA Journal, Vol. 40, No. 4, 2002, pp. 671-680. doi:10.2514/2.1699.

${ }^{37}$ Khavaran, A., "Role of Anisotropy in Turbulent Mixing Noise," AIAA Journal, Vol. 37, No. 7, 1999 , pp. 832-841. doi:10.2514/6.1998-2289.

${ }^{38}$ Leib, S. and Goldstein, M. E., "Hybrid Source Model for Predicting High-Speed Jet Noise," AIAA Journal, Vol. 49, No. 7, 2011, pp. 1324-1335. doi:10.2514/1.J050707.

${ }^{39}$ Keller, J. B., "Geometrical Theory of Diffraction," Journal of the Optical Society of America, Vol. 52, No. 2, 1962, pp. 116-130. doi:10.1364/JOSA.52.000116.

${ }^{40}$ Sommerfeld, A., Lectures on Theoretical Physics: Optics, Vol. 4, Academic Press, Inc, 1964.

${ }^{41}$ Agarwal, A., Dowling, A. P., Shin, H.-C., Graham, W., and Sefi, S., "Ray-Tracing Approach to Calculate Acoustic Shielding by a Flying Wing Airframe," AIAA Journal, Vol. 45, No. 5, 2007, pp. 1080-1090. doi:10.2514/1.26000.

${ }^{42}$ Podboy, G. G., "Jet-Surface Interaction Test: Phased Array Noise Source Localization Results," Proceeding of the ASME Turbo Expo, Copenhagen, Denmark, June 14-18, GT2012-69801, 2012. doi:10.1115/GT2012-69801.

${ }^{43}$ Bridges, J. and Brown, C. A., "Validation of the Small Hot Jet Acoustic Rig for Aeroacoustic Research," 11th AIAA/CEAS Aeroacoustics Conference, AIAA Paper 2005-2846, 2005. doi:10.2514/6.2005-2846.

${ }^{44}$ Anderson, W. K. and Bonhaus, D. L., "An Implicit Upwind Algorithm for Computing Turbulent Flows on Unstructured Grids," Computers and Fluids, Vol. 23, No. 1, 1994, pp. 1-22. doi:10.1016/0045-7930(94)90023-X.

${ }^{45}$ Nielsen, E. J., "Aerodynamic Design Sensitivities on an Unstructured Mesh using the Navier-Stokes Equations and a Discrete Adjoint Formulation," Ph.D. Dissertation, Virginia Polytechnic Institute and State University, 1998.

${ }^{46}$ Menter, F. R., "Two-Equation Eddy-Viscosity Turbulence Models for Engineering Applications," AIAA Journal, Vol. 32, No. 8, 1994, pp. 1598-1605. doi:10.2514/3.12149.

${ }^{47}$ Roe, P. L., "Approximate Riemann Solvers, Parameter Vectors, and Difference Schemes," Journal of Computational Physics, Vol. 135, No. 2, 1981, pp. 357-372. doi:10.1016/0021-9991(81)90128-5.

${ }^{48}$ Roe, P. L., "Characteristic-based schemes for the Euler Equations," Ann. Rev. Fluid Mech., Vol. 18, 1986, pp. 337-365. doi:10.1146/annurev.fl.18.010186.002005.

${ }^{49}$ Smith, M. J. and Miller, S. A. E., "The Effects of Surfaces on the Aerodynamics and Acoustics of Jet Flows," 19th AIAA/CEAS Aeroacoustics Conference (34th AIAA Aeroacoustics Conference), AIAA Paper 2013-2041, 2013. doi:10.2514/6.2013-2041.

${ }^{50}$ Miller, S. A. E., "The Prediction of Noise due to Jet Turbulence Convecting past Flight Vehicle Trailing Edges," AIAA Aviation and Aeronautics Forum and Exposition (20th AIAA/CEAS Aeroacoustics Conference), Atlanta, Georgia, June 16-20, AIAA Paper 2014-2341, 2014. doi:10.2514/6.2014-2341.

${ }^{51}$ Viswanathan, K., "Scaling Laws and a Method for Identifying Components of Jet Noise," AIAA Journal, Vol. 44, No. 10, 2006, pp. 2274-2285. doi:10.2514/1.18486.

${ }^{52}$ Miller, S. A. E., "The Prediction of Broadband Shock-Associated Noise using Reynolds Averaged Navier-Stokes Computational Fluid Dynamics," Ph.D. Dissertation, The Pennsylvania State University University, 2009. 
Figures

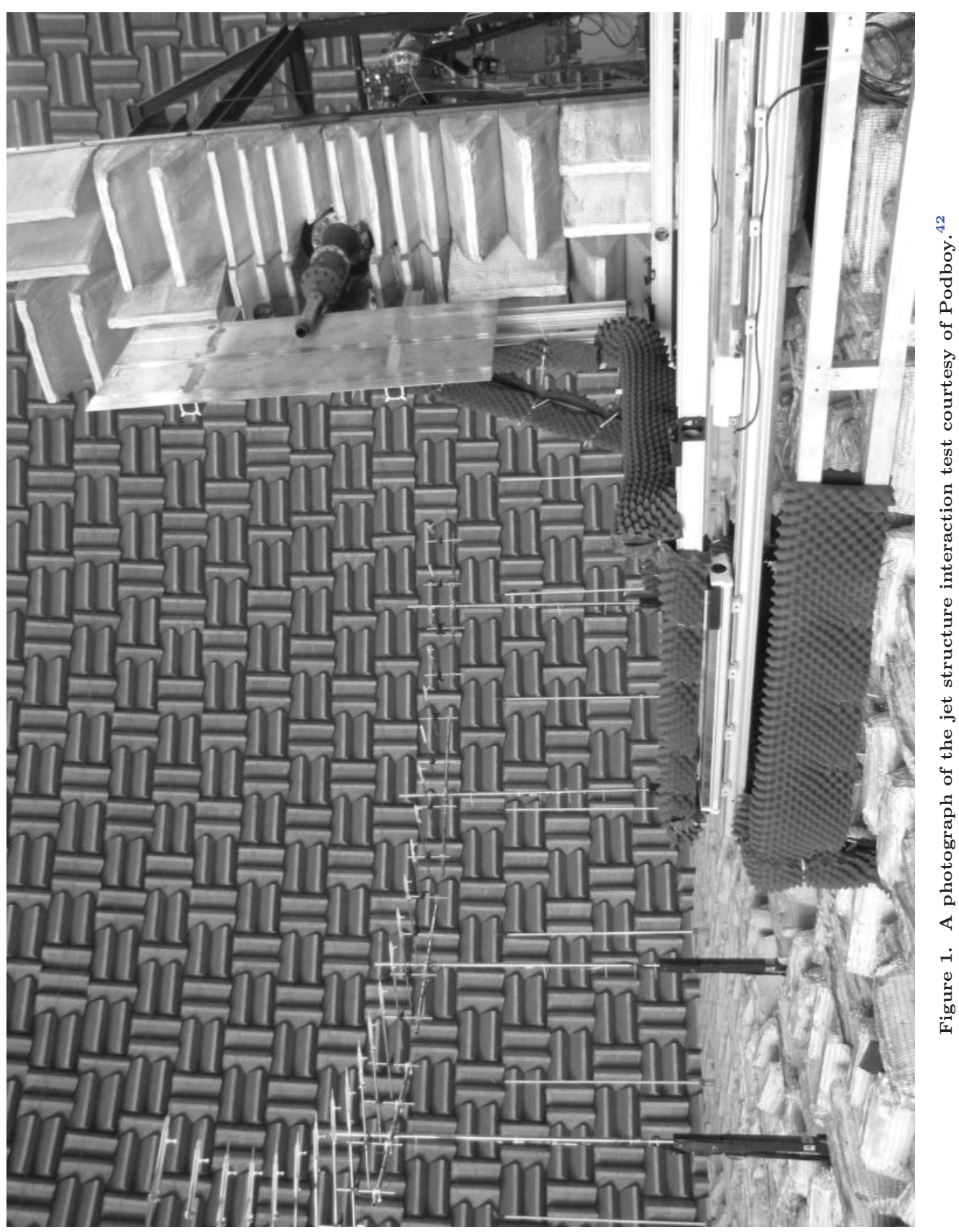




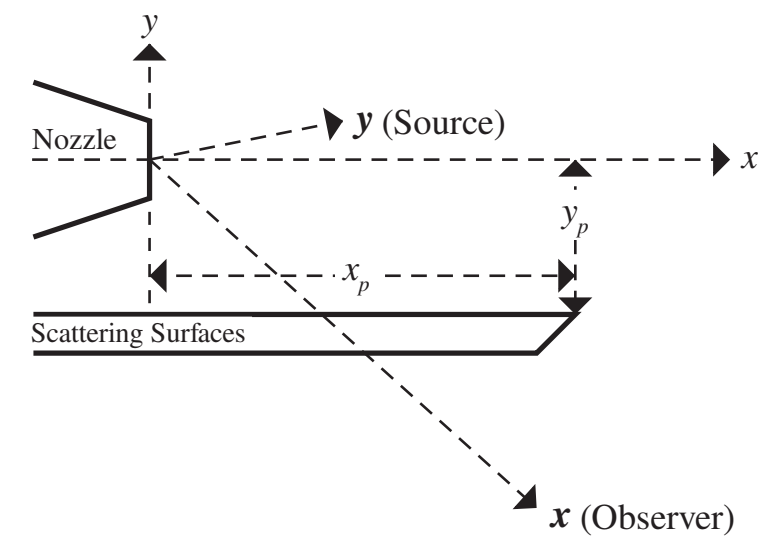

Figure 2. Coordinate system of the jet structure interaction test.

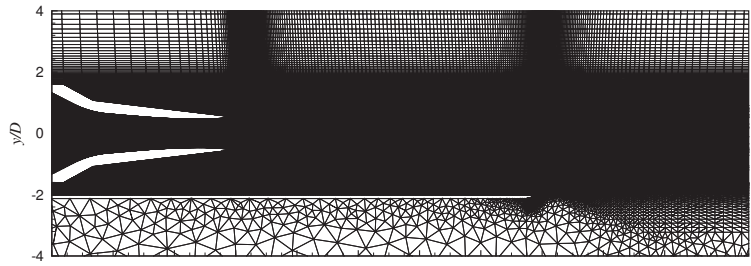

(a) The unstructured-structured computational domain.

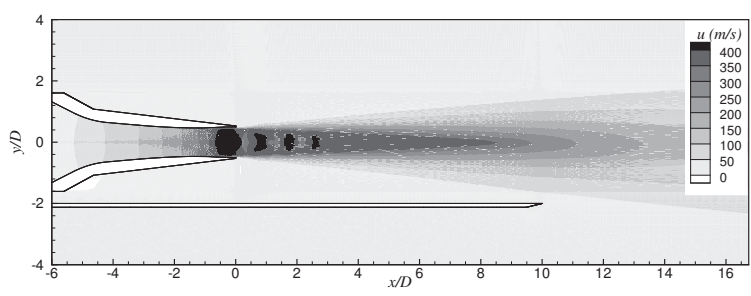

(c) Contours of the streamwise velocity component.

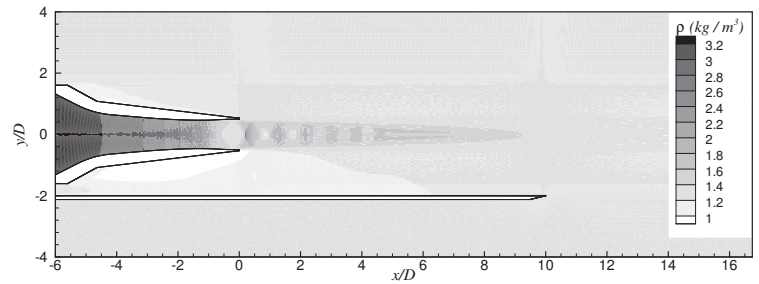

(b) Contours of density.

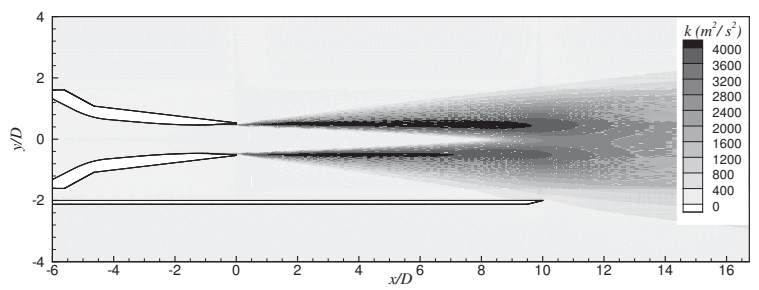

(d) Contours of turbulent kinetic energy.

Figure 3. Quantities of the steady RANS solution on the $x-y$ plane corresponding to the $M_{j}=1.29$ and TTR $=1$ jet from the SMC016 $\left(M_{d}=1.50\right)$ nozzle with $D=0.0508 \mathrm{~m}$. The plate edge is located at $x_{p} / D=10$ and $y_{p} / D=-2$. 


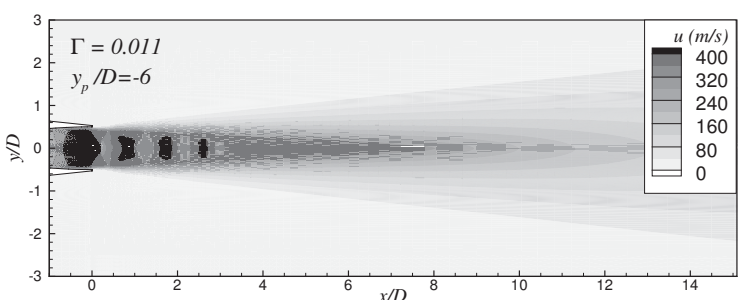

(a) The plate positioned at $y_{p} / D=-6$.

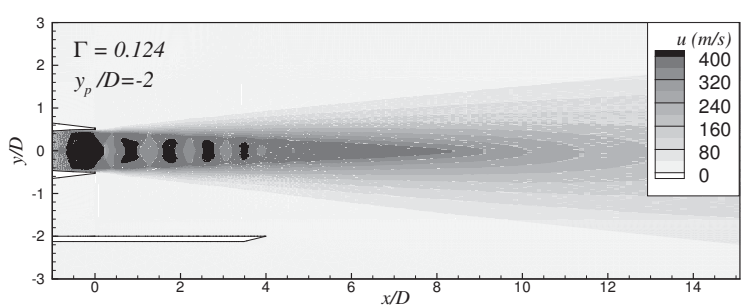

(c) The plate positioned at $y_{p} / D=-2$.

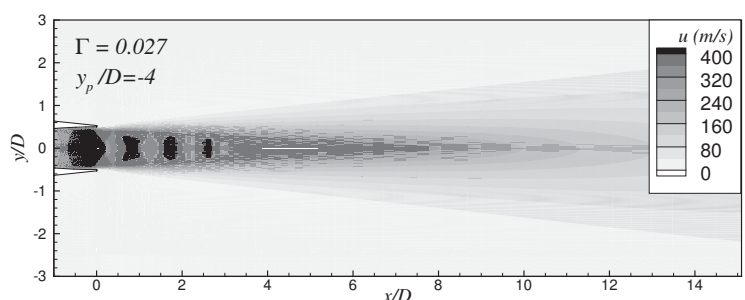

(b) The plate positioned at $y_{p} / D=-4$.

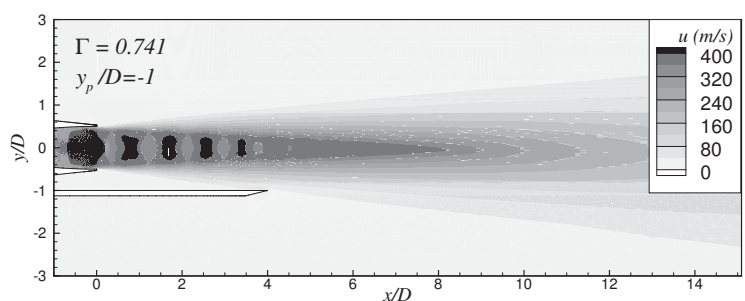

(d) The plate positioned at $y_{p} / D=-1$.

Figure 4. The $M_{d}=1.50$ nozzle operates at $M_{j}=1.29$ and TTR $=1.00$. Contours of the time-averaged streamwise velocity component are shown on the $z=0$ plane. The plate is positioned at $x_{p} / D=4$ and at various radial positions, $y_{p} / D$.

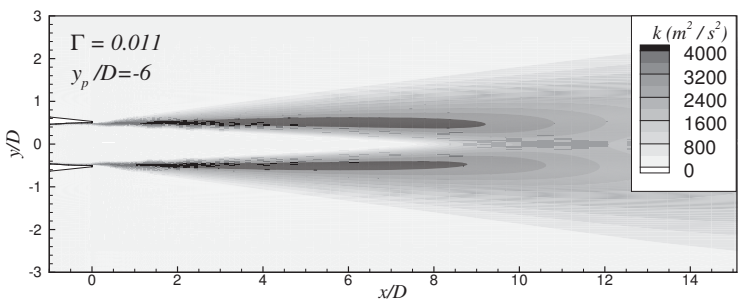

(a) The plate positioned at $y_{p} / D=-6$.

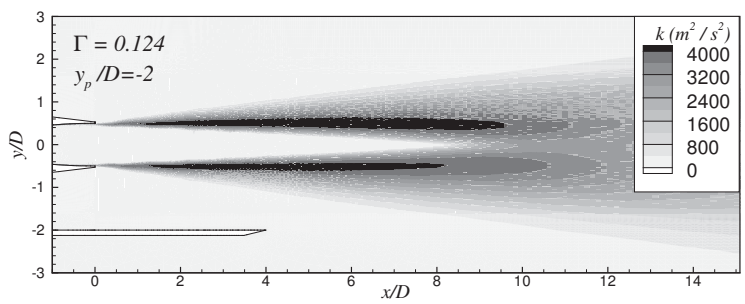

(c) The plate positioned at $y_{p} / D=-2$.

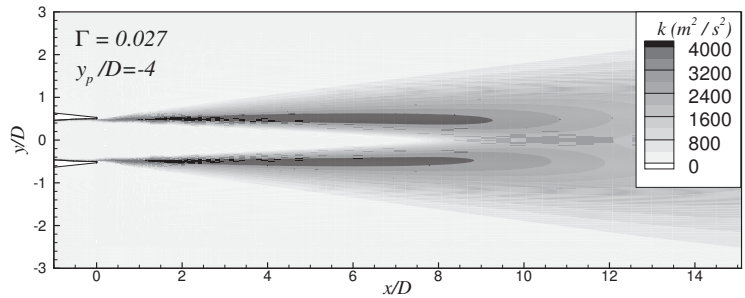

(b) The plate positioned at $y_{p} / D=-4$.

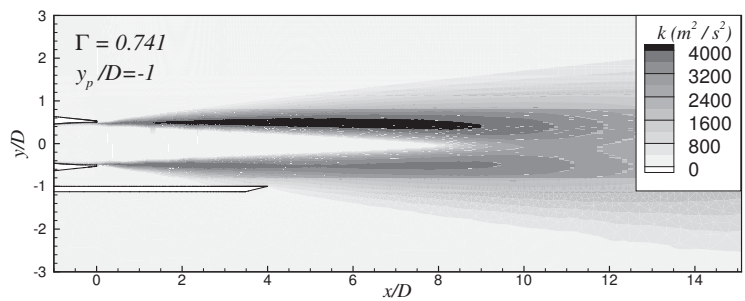

(d) The plate positioned at $y_{p} / D=-1$.

Figure 5. The $M_{d}=1.50$ nozzle operates at $M_{j}=1.29$ and TTR $=1.00$. Contours of turbulent kinetic energy are shown on the $z=0$ plane. The plate is positioned at $x_{p} / D=4$ and at various radial positions, $y_{p} / D$. 


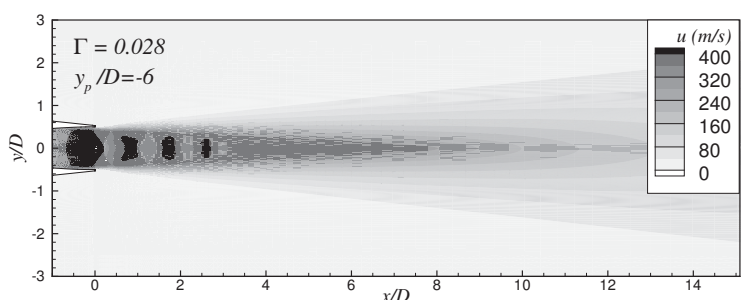

(a) The plate positioned at $y_{p} / D=-6$.

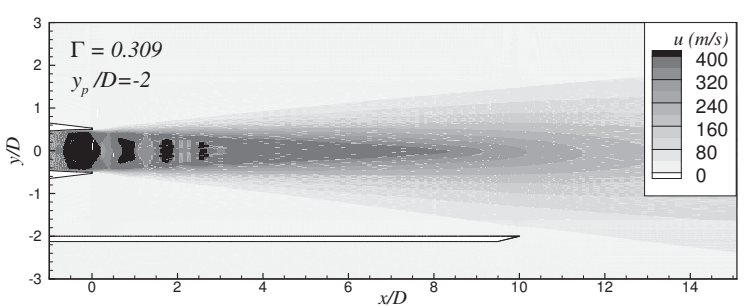

(c) The plate positioned at $y_{p} / D=-2$.

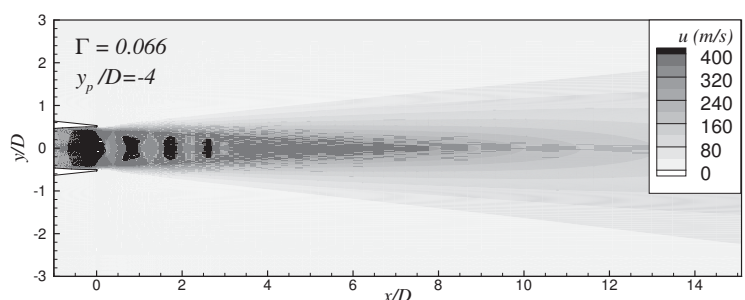

(b) The plate positioned at $y_{p} / D=-4$.

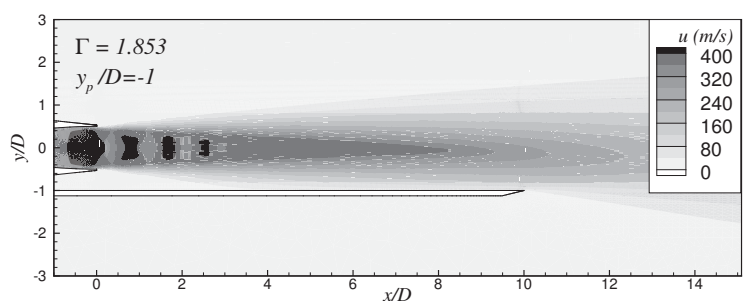

(d) The plate positioned at $y_{p} / D=-1$.

Figure 6. The $M_{d}=1.50$ nozzle operates at $M_{j}=1.29$ and TTR $=1.00$. Contours of the time-averaged streamwise velocity component are shown on the $z=0$ plane. The plate is positioned at $x_{p} / D=10$ and at various radial positions, $y_{p} / D$.

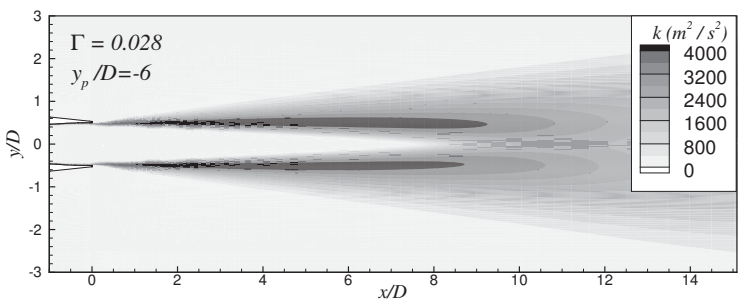

(a) The plate positioned at $y_{p} / D=-6$.

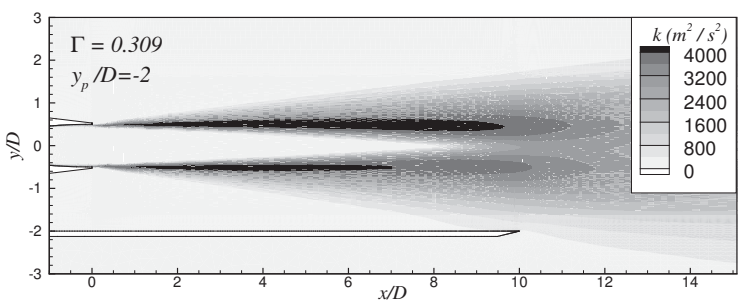

(c) The plate positioned at $y_{p} / D=-2$.

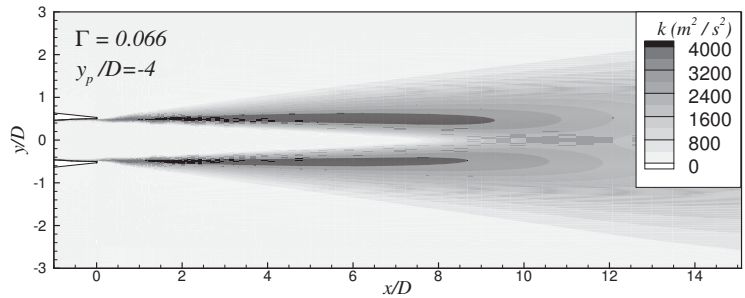

(b) The plate positioned at $y_{p} / D=-4$.

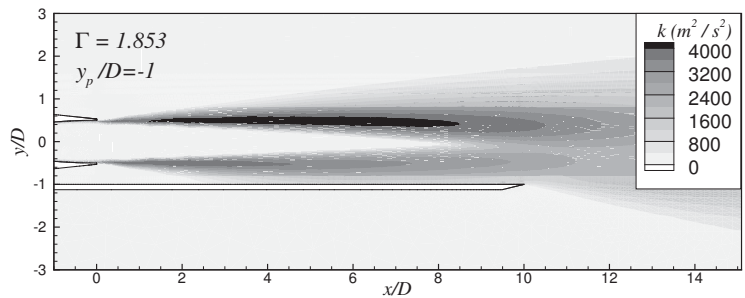

(d) The plate positioned at $y_{p} / D=-1$.

Figure 7. The $M_{d}=1.50$ nozzle operates at $M_{j}=1.29$ and TTR $=1.00$. Contours of turbulent kinetic energy are shown on the $z=0$ plane. The plate is positioned at $x_{p} / D=10$ and at various radial positions, $y_{p} / D$. 


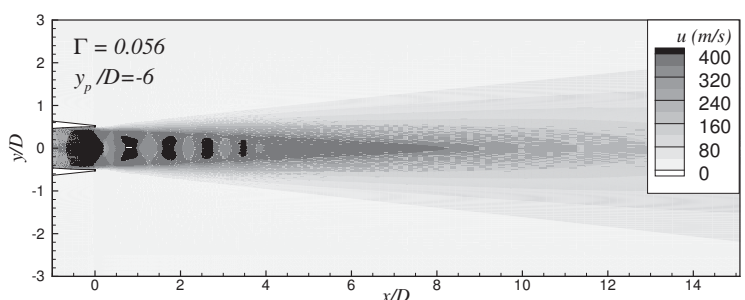

(a) The plate positioned at $y_{p} / D=-6$.

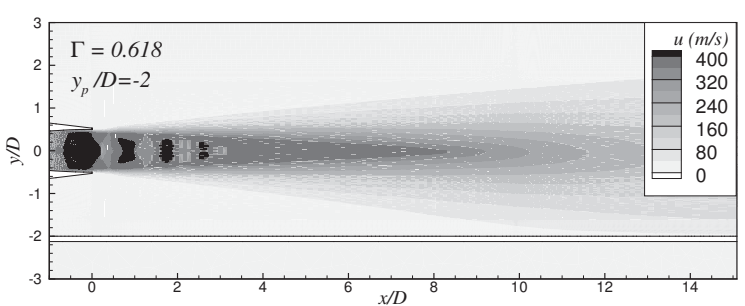

(c) The plate positioned at $y_{p} / D=-2$.

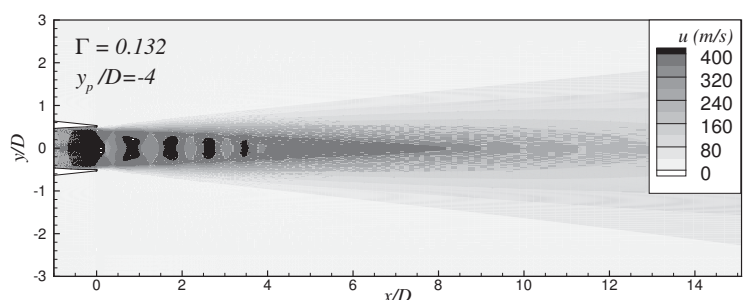

(b) The plate positioned at $y_{p} / D=-4$.

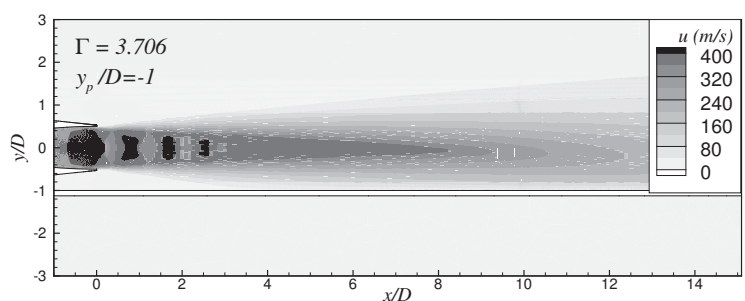

(d) The plate positioned at $y_{p} / D=-1$.

Figure 8. The $M_{d}=1.50$ nozzle operates at $M_{j}=1.29$ and TTR $=1.00$. Contours of the time-averaged streamwise velocity component are shown on the $z=0$ plane. The plate is positioned at $x_{p} / D=20$ and at various radial positions, $y_{p} / D$.

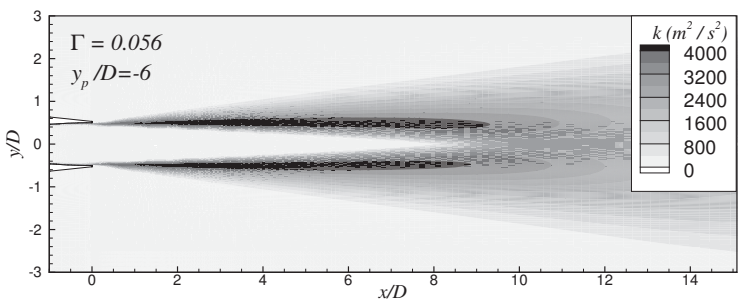

(a) The plate positioned at $y_{p} / D=-6$.

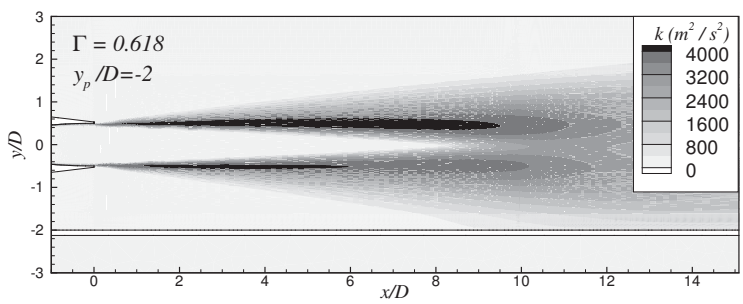

(c) The plate positioned at $y_{p} / D=-2$.

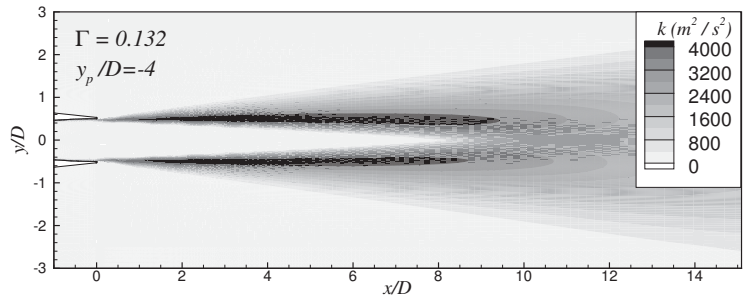

(b) The plate positioned at $y_{p} / D=-4$.

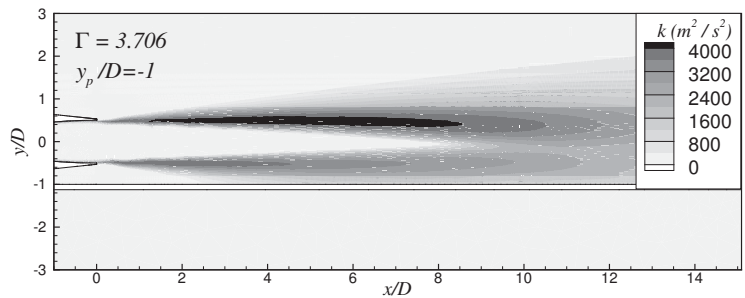

(d) The plate positioned at $y_{p} / D=-1$.

Figure 9. The $M_{d}=1.50$ nozzle operates at $M_{j}=1.29$ and TTR $=1.00$. Contours of turbulent kinetic energy are shown on the $z=0$ plane. The plate is positioned at $x_{p} / D=20$ and at various radial positions, $y_{p} / D$. 

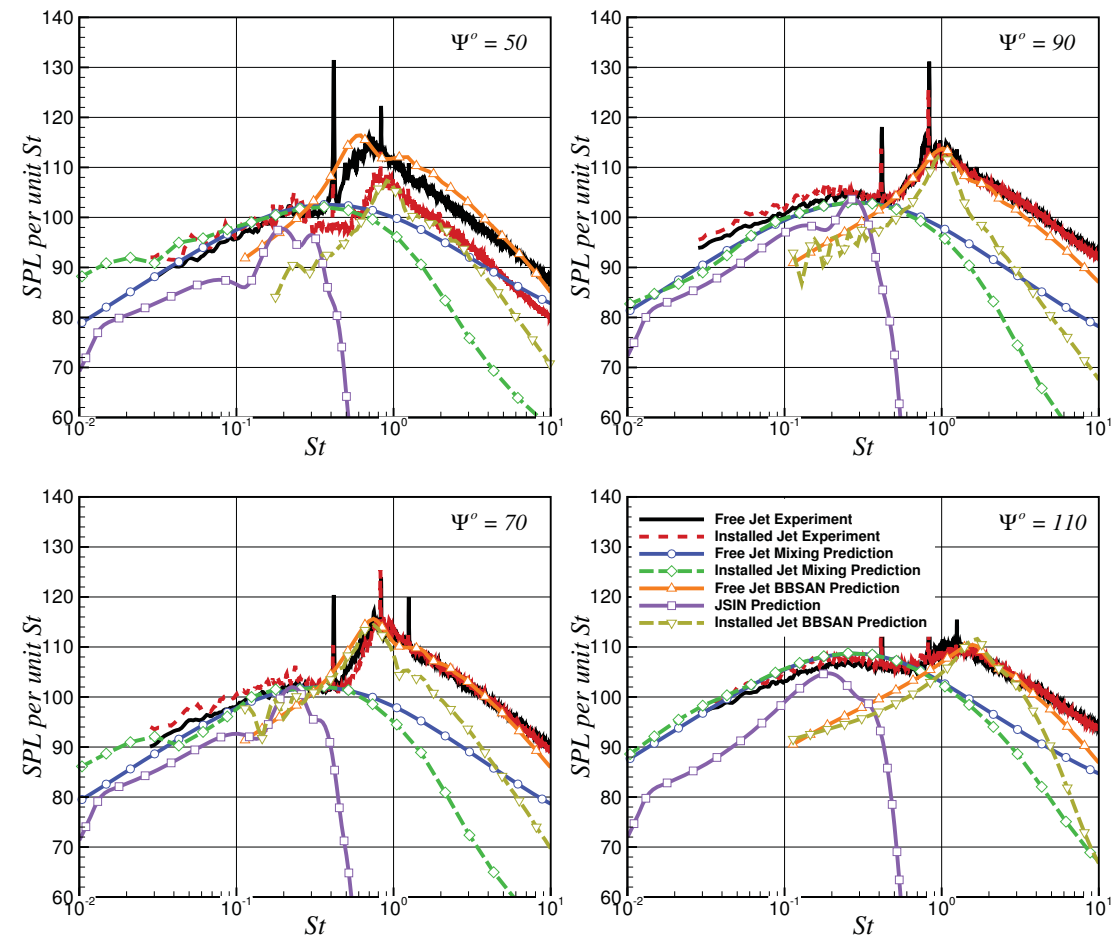

Figure 10. Predictions compared with measurement for the $M_{j}=1.29$ and TTR $=1$ jet from the SMC016 nozzle with $D=0.0508 \mathrm{~m}$ at $R / D=100$ and varying angle $\Psi$. The plate is located at $x_{p} / D=4$ and $y_{p} / D=-2$. These conditions correspond to $\Gamma=0.124$.
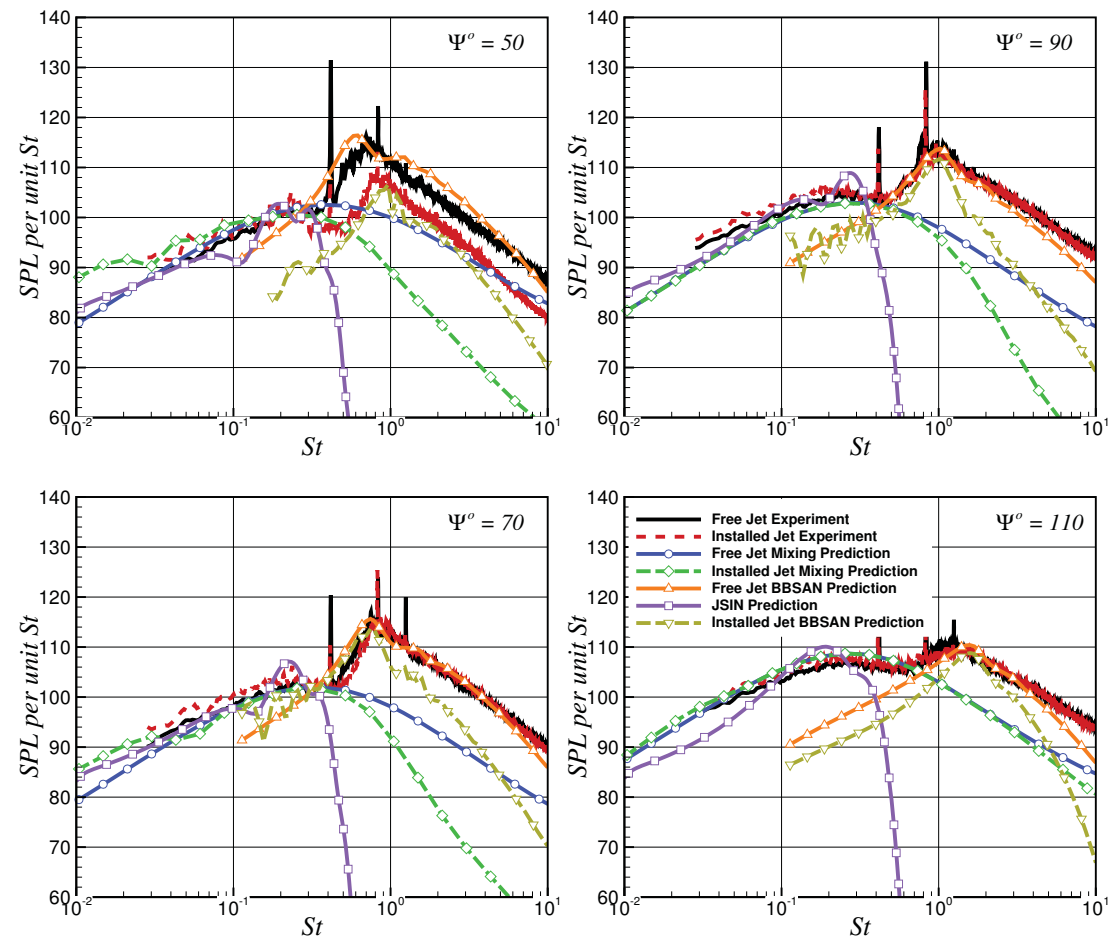

Figure 11. Predictions compared with measurement for the $M_{j}=1.29$ and TTR $=1$ jet from the SMC016 nozzle with $D=0.0508 \mathrm{~m}$ at $R / D=100$ and varying angle $\Psi$. The plate is located at $x_{p} / D=4$ and $y_{p} / D=-4$. These conditions correspond to $\Gamma=0.027$. 

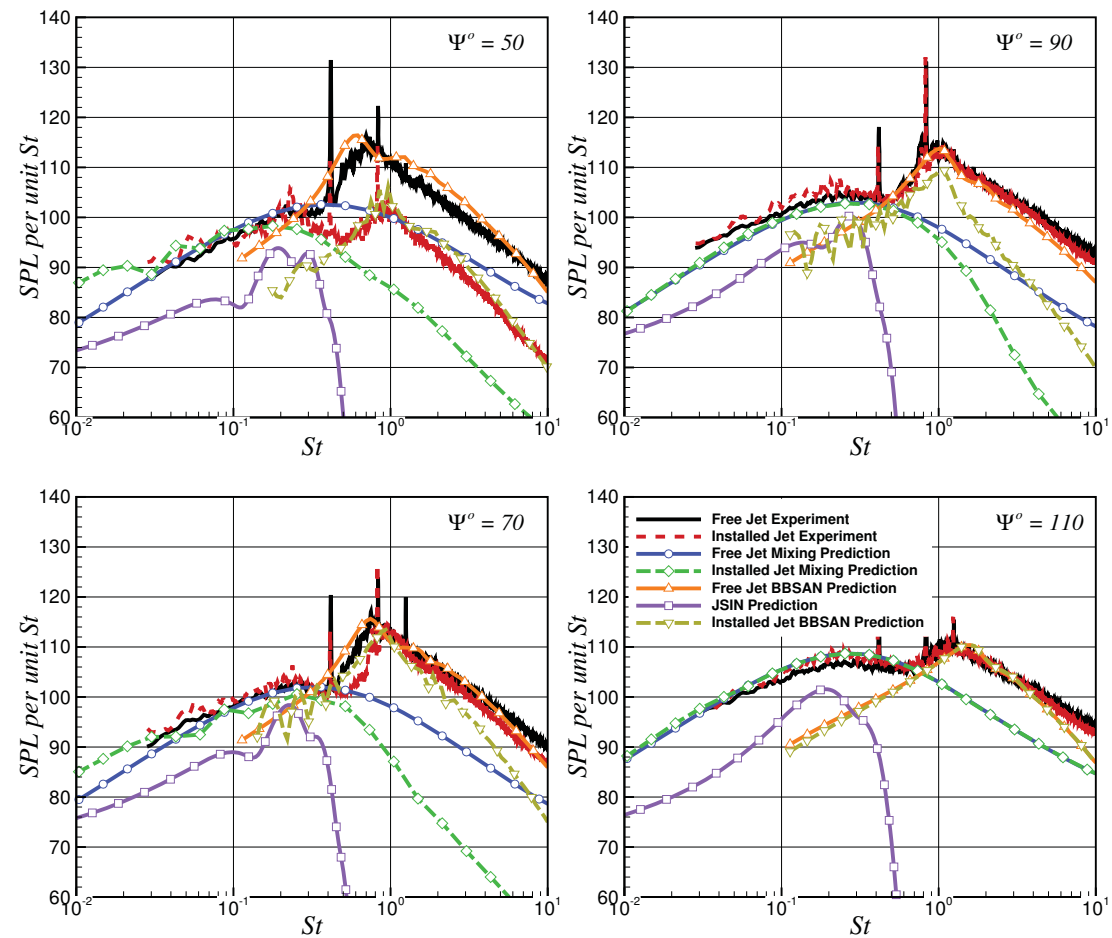

Figure 12. Predictions compared with measurement for the $M_{j}=1.29$ and TTR $=1$ jet from the SMC016 nozzle with $D=0.0508 \mathrm{~m}$ at $R / D=100$ and varying angle $\Psi$. The plate is located at $x_{p} / D=4$ and $y_{p} / D=-6$. These conditions correspond to $\Gamma=0.011$.
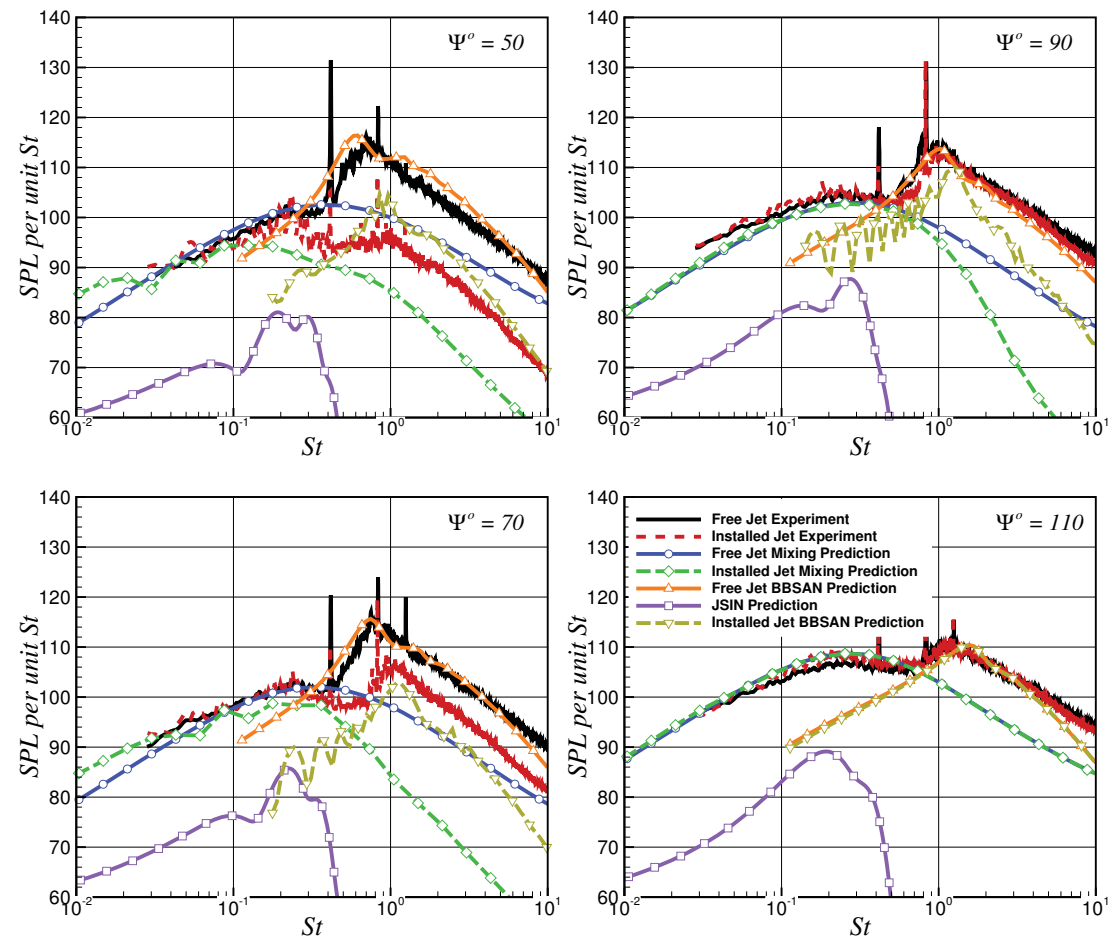

Figure 13. Predictions compared with measurement for the $M_{j}=1.29$ and TTR $=1$ jet from the SMC016 nozzle with $D=0.0508 \mathrm{~m}$ at $R / D=100$ and varying angle $\Psi$. The plate is located at $x_{p} / D=4$ and $y_{p} / D=-8$. These conditions correspond to $\Gamma=0.006$. 

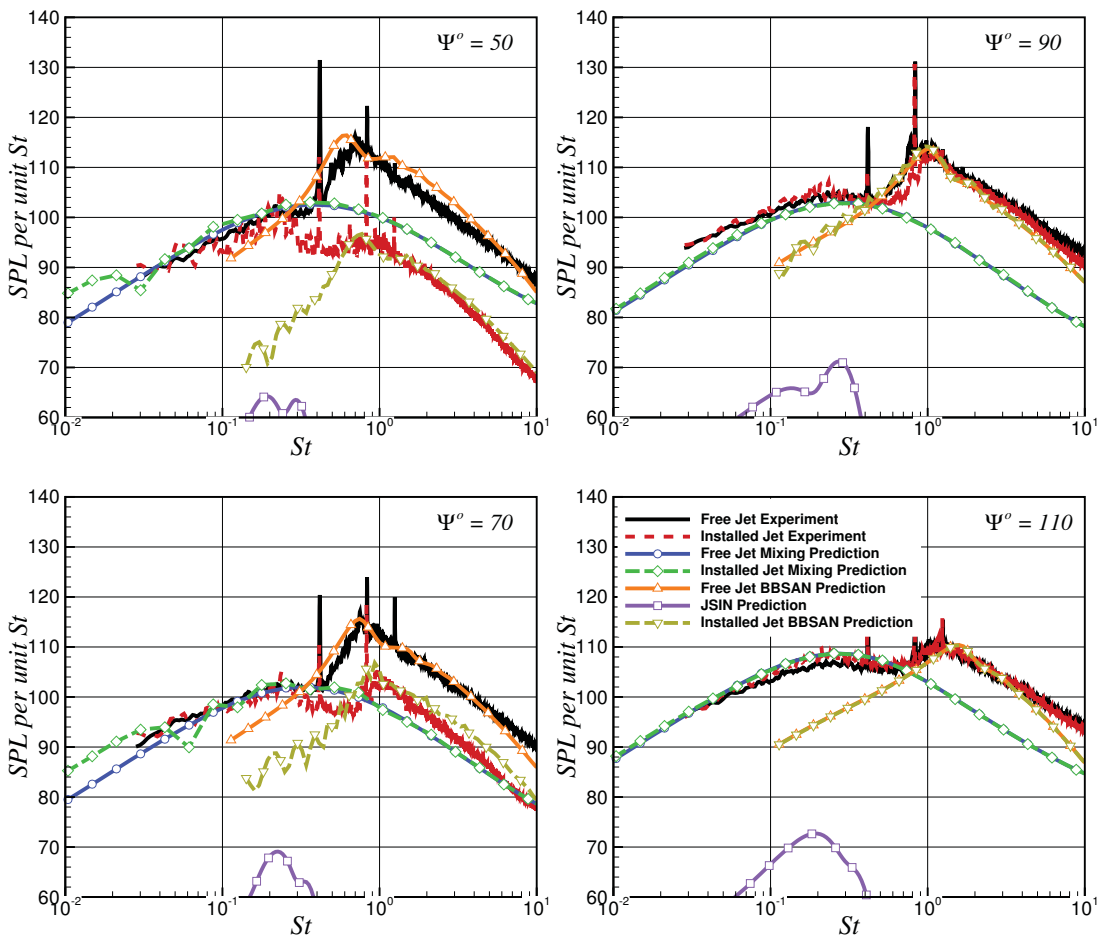

Figure 14. Predictions compared with measurement for the $M_{j}=1.29$ and TTR $=1$ jet from the SMC016 nozzle with $D=0.0508 \mathrm{~m}$ at $R / D=100$ and varying angle $\Psi$. The plate is located at $x_{p} / D=4$ and $y_{p} / D=-10$. These conditions correspond to $\Gamma=0.004$.
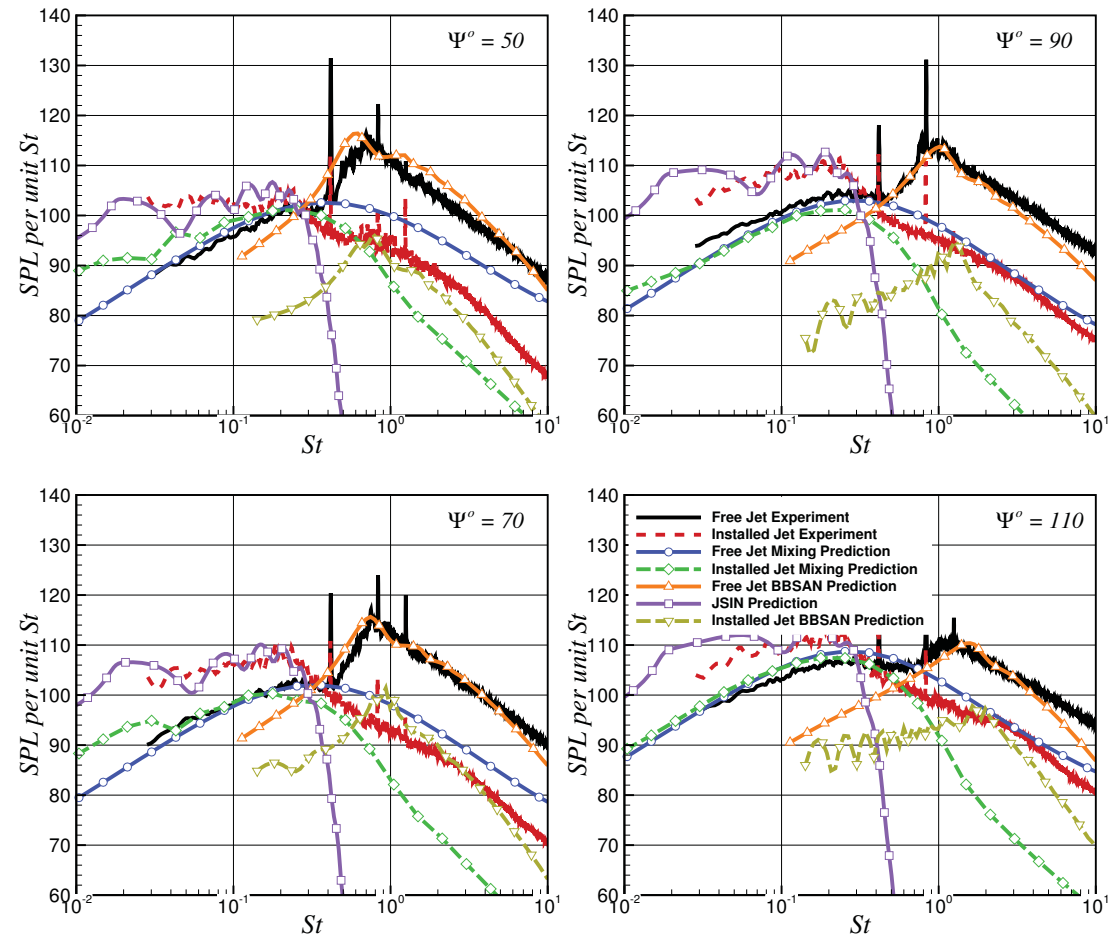

Figure 15. Predictions compared with measurement for the $M_{j}=1.29$ and TTR $=1$ jet from the SMC016 nozzle with $D=0.0508 \mathrm{~m}$ at $R / D=100$ and varying angle $\Psi$. The plate is located at $x_{p} / D=10$ and $y_{p} / D=-2$. These conditions correspond to $\Gamma=0.309$. 

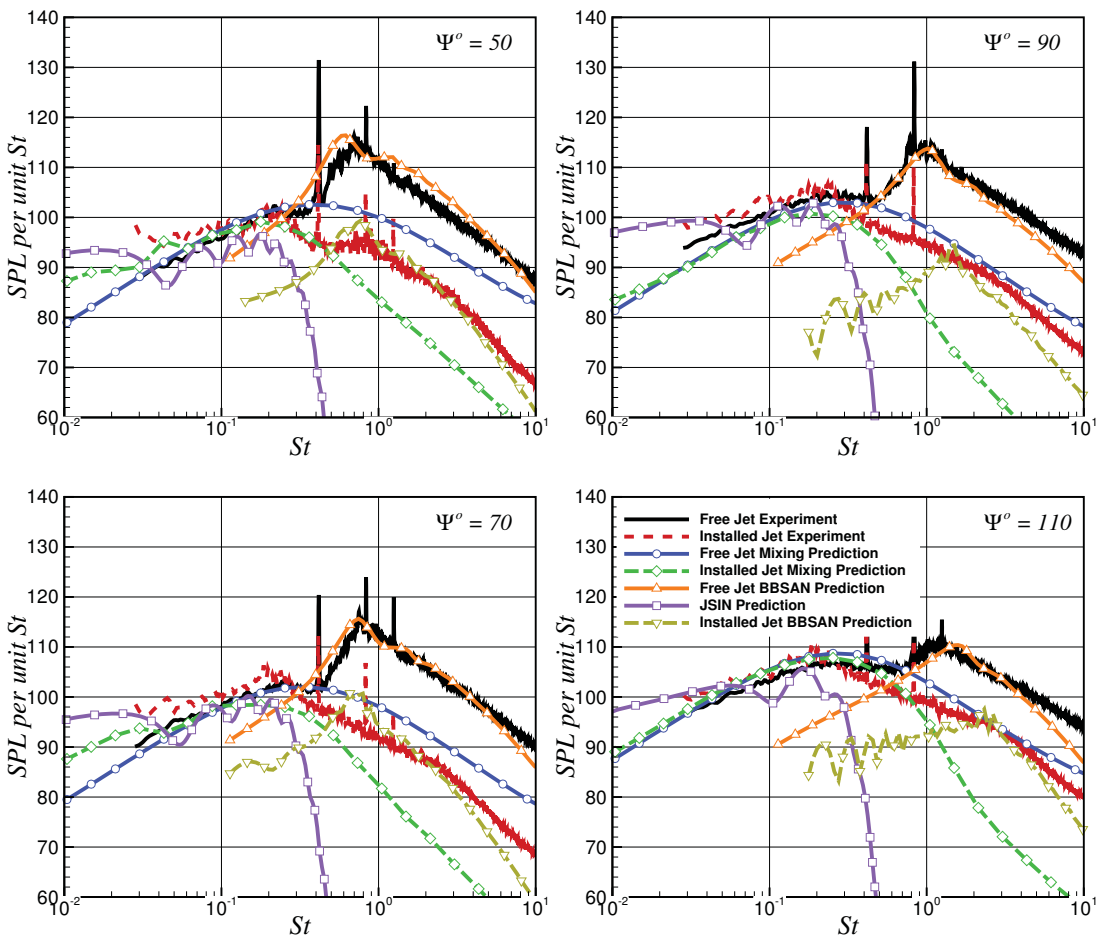

Figure 16. Predictions compared with measurement for the $M_{j}=1.29$ and TTR $=1$ jet from the SMC016 nozzle with $D=0.0508 \mathrm{~m}$ at $R / D=100$ and varying angle $\Psi$. The plate is located at $x_{p} / D=10$ and $y_{p} / D=-4$. These conditions correspond to $\Gamma=0.066$.
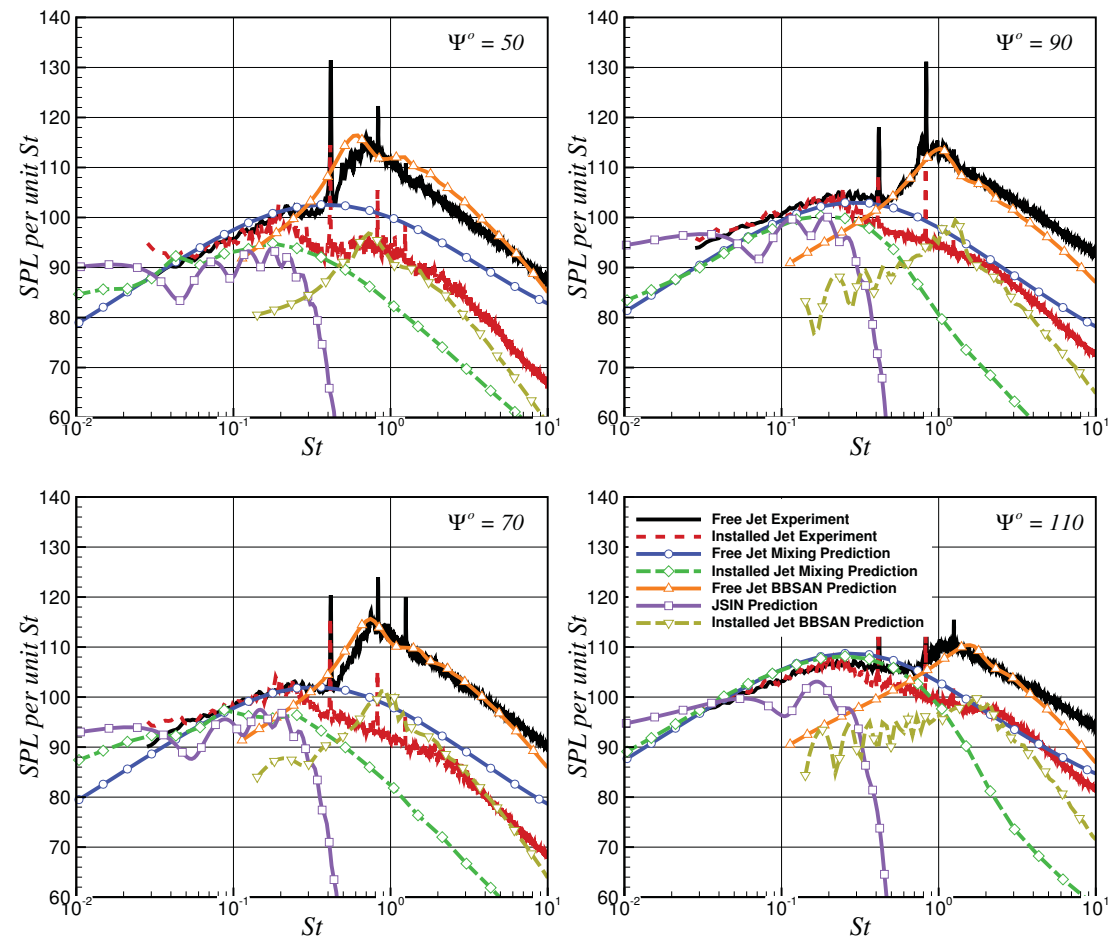

Figure 17. Predictions compared with measurement for the $M_{j}=1.29$ and TTR $=1$ jet from the SMC016 nozzle with $D=0.0508 \mathrm{~m}$ at $R / D=100$ and varying angle $\Psi$. The plate is located at $x_{p} / D=10$ and $y_{p} / D=-6$. These conditions correspond to $\Gamma=0.028$. 

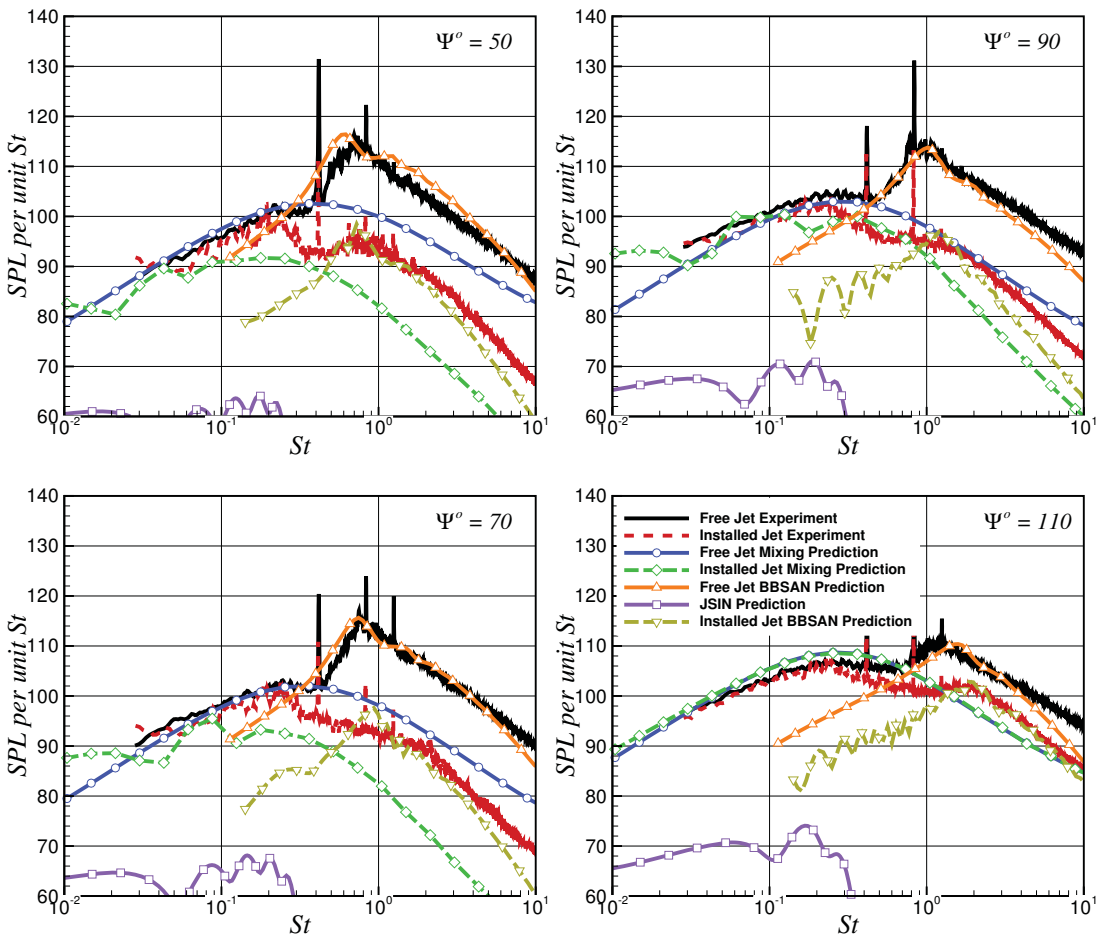

Figure 18. Predictions compared with measurement for the $M_{j}=1.29$ and TTR $=1$ jet from the SMC016 nozzle with $D=0.0508 \mathrm{~m}$ at $R / D=100$ and varying angle $\Psi$. The plate is located at $x_{p} / D=10$ and $y_{p} / D=-10$. These conditions correspond to $\Gamma=0.010$.
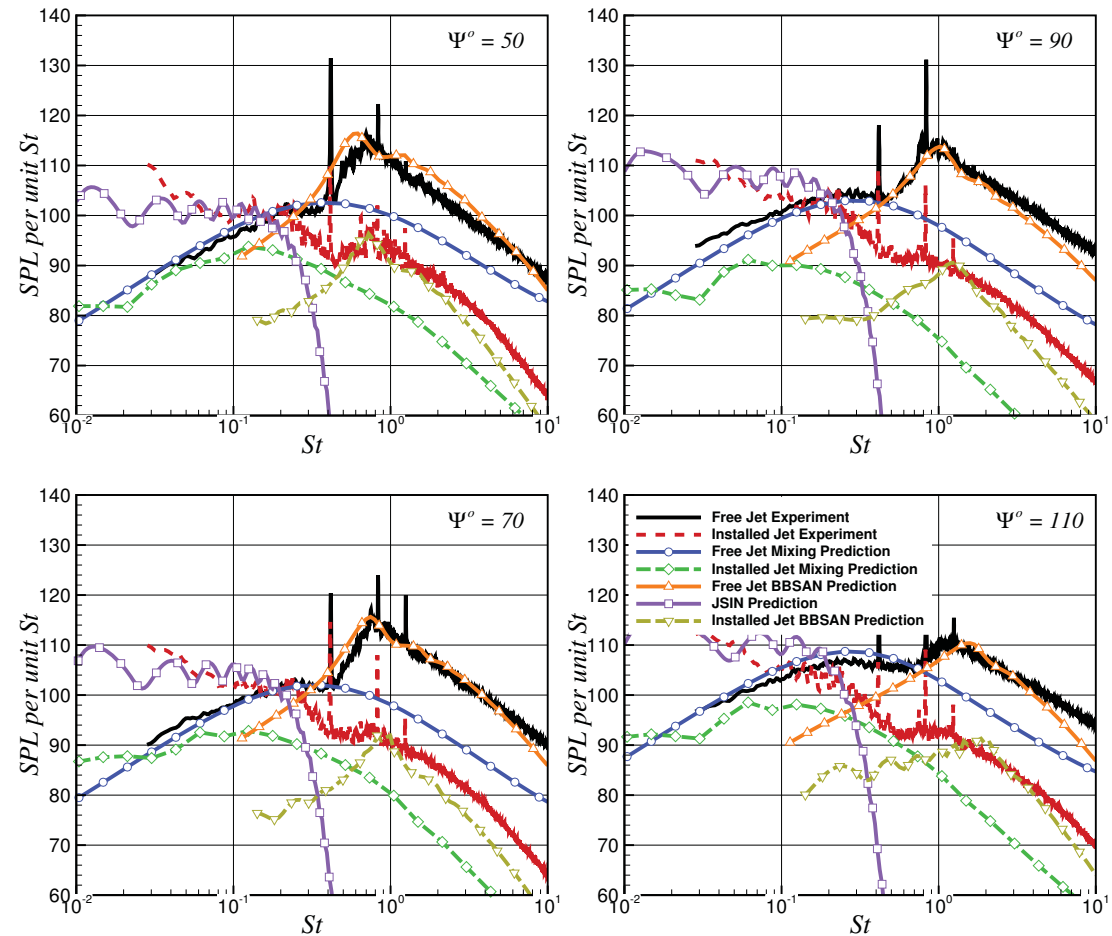

Figure 19. Predictions compared with measurement for the $M_{j}=1.29$ and TTR $=1$ jet from the SMC016 nozzle with $D=0.0508 \mathrm{~m}$ at $R / D=100$ and varying angle $\Psi$. The plate is located at $x_{p} / D=20$ and $y_{p} / D=-2$. These conditions correspond to $\Gamma=0.618$. 

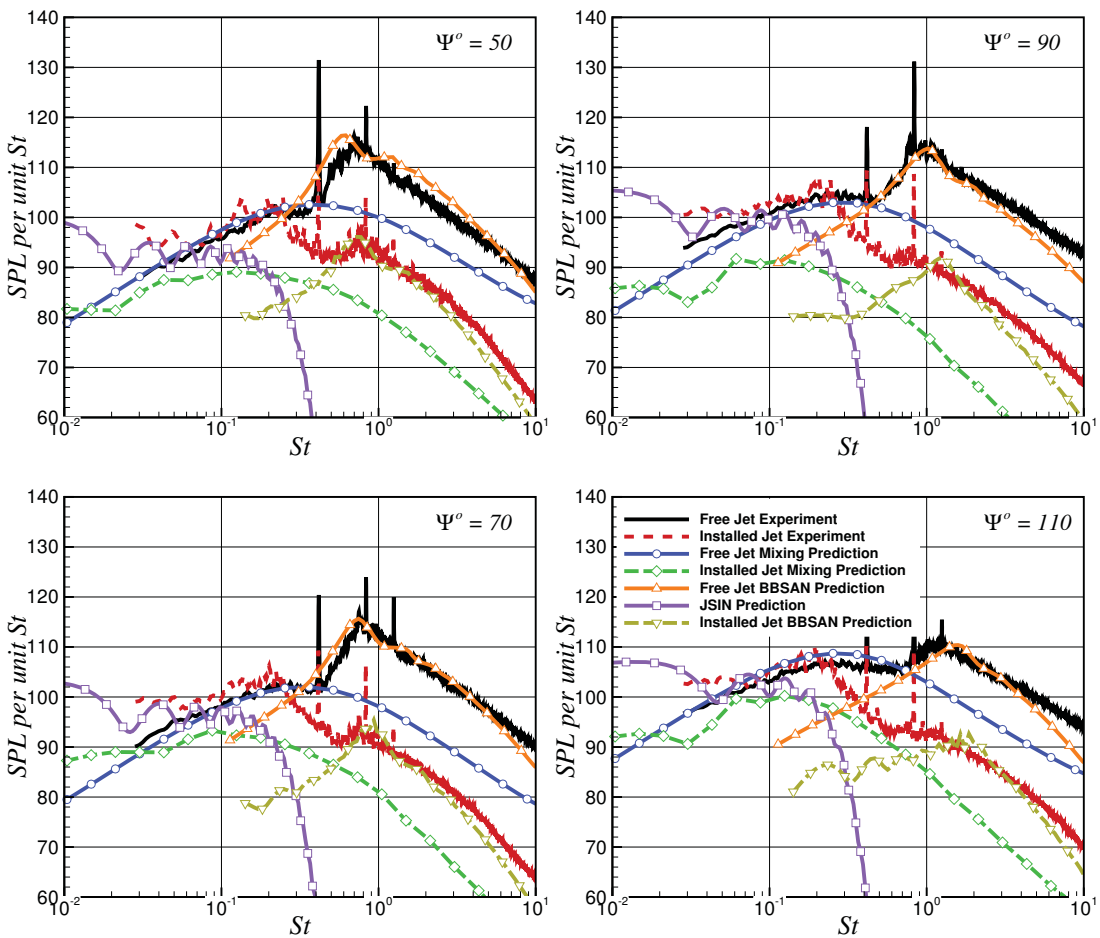

Figure 20. Predictions compared with measurement for the $M_{j}=1.29$ and TTR $=1$ jet from the SMC016 nozzle with $D=0.0508 \mathrm{~m}$ at $R / D=100$ and varying angle $\Psi$. The plate is located at $x_{p} / D=20$ and $y_{p} / D=-6$. These conditions correspond to $\Gamma=0.056$.
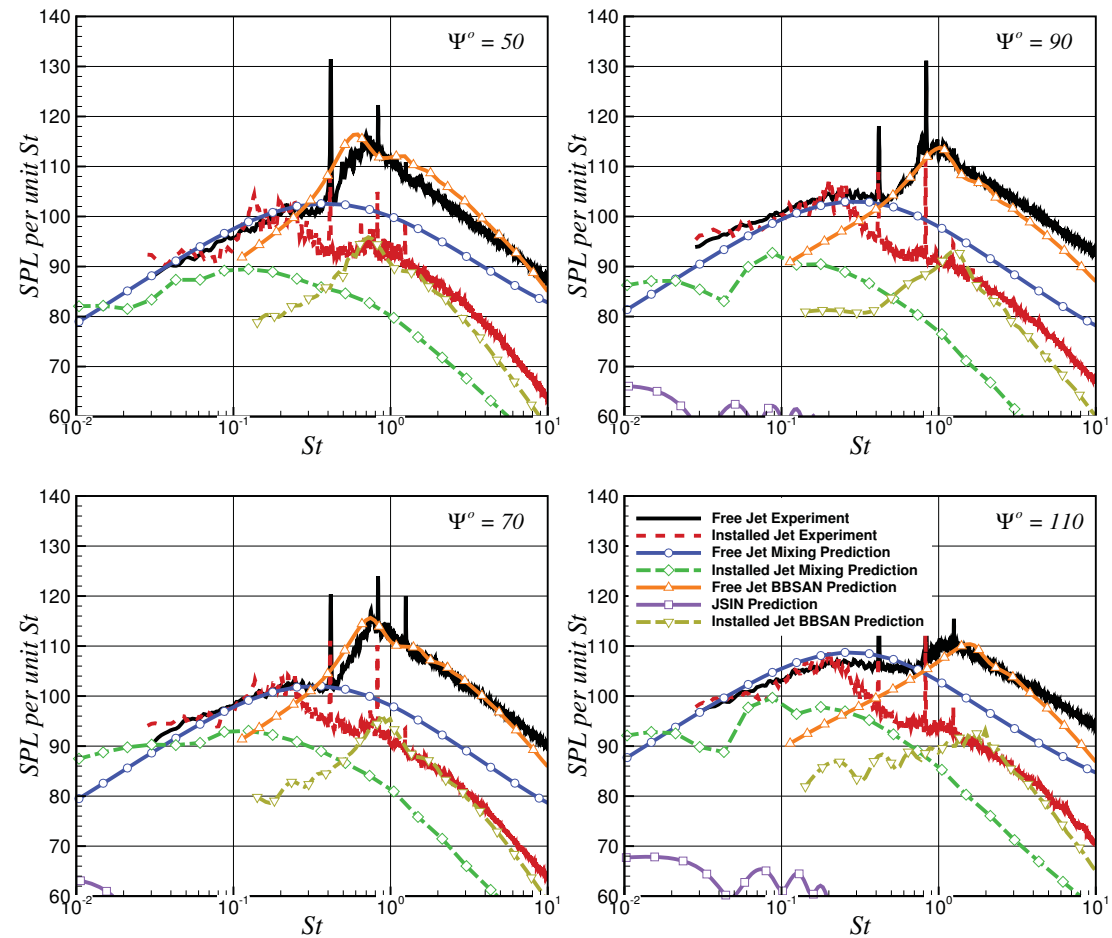

Figure 21. Predictions compared with measurement for the $M_{j}=1.29$ and TTR $=1$ jet from the SMC016 nozzle with $D=0.0508 \mathrm{~m}$ at $R / D=100$ and varying angle $\Psi$. The plate is located at $x_{p} / D=20$ and $y_{p} / D=-10$. These conditions correspond to $\Gamma=0.020$. 